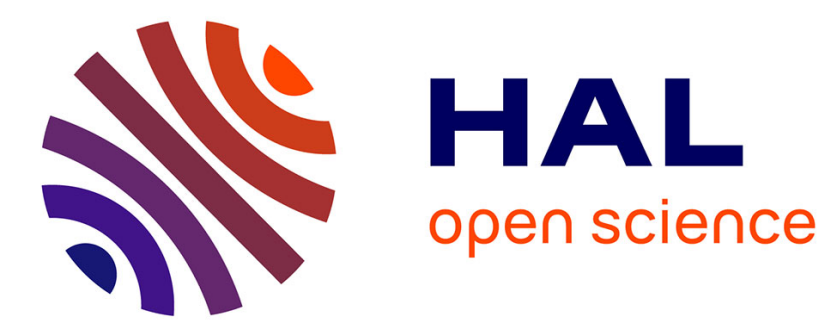

\title{
Iterated variable neighborhood search for the capacitated clustering problem
}

\author{
Xiangjing Lai, Jin-Kao Hao
}

\section{To cite this version:}

Xiangjing Lai, Jin-Kao Hao. Iterated variable neighborhood search for the capacitated clustering problem. Engineering Applications of Artificial Intelligence, 2016, 56, pp.102-120. 10.1016/j.engappai.2016.08.004 . hal-01412523v2

HAL Id: hal-01412523

https://hal.science/hal-01412523v2

Submitted on 4 Jun 2017

HAL is a multi-disciplinary open access archive for the deposit and dissemination of scientific research documents, whether they are published or not. The documents may come from teaching and research institutions in France or abroad, or from public or private research centers.
L'archive ouverte pluridisciplinaire HAL, est destinée au dépôt et à la diffusion de documents scientifiques de niveau recherche, publiés ou non, émanant des établissements d'enseignement et de recherche français ou étrangers, des laboratoires publics ou privés. 


\title{
Iterated variable neighborhood search for the capacitated clustering problem
}

\author{
Xiangjing Lai ${ }^{\mathrm{a}}$ and Jin-Kao Hao ${ }^{\mathrm{a}, \mathrm{b}, *}$, \\ ${ }^{a}$ LERIA, Université d'Angers, 2 Boulevard Lavoisier, 49045 Angers, France \\ ${ }^{\mathrm{b}}$ Institut Universitaire de France, Paris, France \\ Engineering Applications of Artificial Intelligence \\ http://dx.doi.org/10.1016/j.engappai.2016.08.004
}

\begin{abstract}
The NP-hard capacitated clustering problem (CCP) is a general model with a number of relevant applications. This paper proposes a highly effective iterated variable neighborhood search (IVNS) algorithm for solving the problem. IVNS combines an extended variable neighborhood descent method and a randomized shake procedure to explore effectively the search space. The computational results obtained on three sets of 133 benchmarks reveal that the proposed algorithm competes favorably with the state-of-the-art algorithms in the literature both in terms of solution quality and computational efficiency. In particular, IVNS discovers an improved best known result (new lower bounds) for 28 out of 83 most popular instances, while matching the current best known results for the remaining 55 instances. Several essential components of the proposed algorithm are investigated to understand their impacts on the performance of algorithm.
\end{abstract}

Keywords: Capacitated clustering; grouping problem; variable neighborhood search; heuristics.

\section{$1 \quad 1$ Introduction}

2 Given a weighted undirect graph $G=(V, E, C, w)$, where $V=\left\{v_{1}, v_{2}, \ldots, v_{n}\right\}$ 3 is the set of $n$ nodes, $E$ is the set of its edges, $C=\left\{c_{i j}:\left\{v_{i}, v_{j}\right\} \in E\right\}$ 4 represents the set of edge weights, and $w=\left\{w_{i} \geq 0: v_{i} \in V\right\}$ is the set 5 of node weights, the capacitated clustering problem (CCP) is to partition the

* Corresponding author.

Email addresses: laixiangjing@gmail.com (Xiangjing Lai), hao@info. univ-angers.fr (Jin-Kao Hao). 
node set $V$ into a fixed number $p$ ( $p \leq n$ is given) of disjoint clusters (or groups) such that the sum of node weights of each cluster lies in a given interval $[L, U]$ while maximizing the sum of the edge weights whose two associated endpoints locate in the same cluster. In some related literature like [9,23], an edge weight $c_{i j} \in C$ is also called the benefit of the edge $\left\{v_{i}, v_{j}\right\}$, while $L$ and $U$ are called the lower and upper capacity limits of a cluster.

Formally, the CCP can be expressed as the following quadratic program with binary variables $X_{i g}$ taking the value of 1 if node $v_{i}$ is in cluster $g$ and 0 otherwise $[9,23]$ :

$$
\begin{gathered}
(C C P) \quad \text { Maximize } \sum_{g=1}^{p} \sum_{i=1}^{n-1} \sum_{j=i+1}^{n} c_{i j} X_{i g} X_{j g} \\
\text { Subject to } \sum_{g=1}^{p} X_{i g}=1, i=1,2, \ldots, n \\
L \leq \sum_{i=1}^{n} w_{i} X_{i g} \leq U, g=1,2, \ldots, p \\
X_{i g} \in\{0,1\}, i=1,2, \ldots, n ; g=1,2, \ldots, p \\
c_{i j}=0, \forall\left\{v_{i}, v_{j}\right\} \notin E
\end{gathered}
$$

where the set of constraints (2) guarantees that each node is located in exactly one cluster (or group) and the set of constraints (3) forces the sum of node weights of each cluster to be at least $L$ and at most $U$. The set of constraints (5) ensures that the benefit between nodes $v_{i}$ and $v_{j}$ is 0 if $\left\{v_{i}, v_{j}\right\} \notin E$.

The CCP is closely related to three other clustering problems: the graph partitioning problem (GPP) $[2-4,15,30]$, the maximally diverse grouping problem (MDGP) [5,10,14,19,28,29,33], and the handover minimization problem (HMP) $[23,26]$. First, the GPP is a special case of the CCP when the lower and upper capacity limits of the clusters are respectively set to 0 and $(1+$ $\epsilon)\left\lceil\frac{\sum_{i=1}^{n} w_{i}}{p}\right\rceil$, where $\epsilon(\in[0,1))$ is a predetermined imbalance parameter. As such, the CCP is also known as the node capacitated graph partitioning problem $[11-13,27]$ or the min-cut clustering problem [20] in the literature. Second, the MDGP is also a special case of the CCP when the given graph is a complete graph and the nodes have a unit weight $\left(w_{i}=1, i=1,2, \ldots, n\right)$ [23]. Additionally, as discussed in [23,26], the HMP can be viewed as a practical application of the CCP in the context of mobile networks.

Given that the CCP generalizes the NP-hard MDPG, GPP, and HMP problems, the CCP is at least as computationally difficult as these problems. Moreover, any real-world applications that can be formulated by the MDPG, GPP, or HMP models can be cast as the CCP, such as creation of peer review groups 
[7], parallel computing [18], assignment of students to groups [19], VLSI design [33], etc.

Given the NP-hard nature of the CCP and its practical importance, a large number of studies have been proposed to investigate the problem and the three related clustering problems. Below, we highlight some most recent approaches on the CCP, while refereeing the reader to two recent papers $[9,23]$ for a comprehensive review of existing studies in the literature.

In 2011, Deng and Bard [9] proposed a greedy randomized adaptive search procedure with path relinking (GRASP $+\mathrm{PR}$ ) by hybridizing a construction procedure of initial solution, a randomized variable neighborhood descent method as well as a path-relinking procedure. The reported computational results showed that the proposed GRASP $+\mathrm{PR}$ algorithm outperforms the reference algorithms. In 2013, Morán-Mirabal et al. [26] proposed the following three heuristic algorithms for the HMP problem that is a special case of the CCP: a GRASP method (denoted by GQAP in their paper), an evolutionary path-relinking algorithm combined with GRASP (GevPR-HMP), and a population-based biased random-key genetic algorithm (BRKGA). Their study showed that GevPR-HMP achieved the best performance among the three proposed algorithms. In 2014, Lewis et al. [22] made a comparison between the linear and nonlinear models for the CCP under the framework of exact methods, and showed that the quadratic model generally outperforms its equivalent linear alternatives.

Recently (2015), Martínez-Gavara et al. [23] introduced several heuristic algorithms for the CCP, including a new GRASP method, a tabu search (TS) method, and a hybrid algorithm combining the proposed GRASP and tabu search methods (GRASP + TS). The authors also adapted a tabu search algorithm with strategic oscillation (TS_SO) originally designed for the MDGP to solve the CCP. Their study showed that the proposed GRASP + TS and TS algorithms significantly outperform their reference algorithms, including their GRASP method, Deng and Bard's GRASP and TS SO presented in [9], as well as the GevPR-HMP algorithm of [26]. Consequently, the TS and GRASP + TS algorithms proposed in [23] can be considered as the current best performing approaches for the CCP.

In this paper, we are interested in solving the general CCP problem approximately and propose for this purpose an effective iterated variable neighborhood search algorithm (IVNS). The main contributions of the present work can be highlighted as follows:

- The proposed IVNS algorithm introduces an extended variable neighborhood descent (EVND) method to ensure an intensified local optimization. Contrary to the standard variable neighborhood descent (VND) method 
[24], our EVND method focuses on a more balanced exploitation between different neighborhoods, which provides the search with a reinforced diversification effect. Additionally, IVNS integrates two dedicated construction procedures to generate initial solutions and a randomized shake procedure to escape deep local optima (i.e., local optimum solutions which are difficult to attain and difficult to escape for a search algorithm).

- When it is assessed on three sets of 133 benchmark instances of the literature, the proposed IVNS algorithm achieves highly competitive performances both in terms of the solution quality and computational efficiency compared to the state-of-the-art results. On the two sets of 50 standard instances, IVNS outperforms the state-of-the-art CCP algorithms in the literature. Moreover, for the 83 popular benchmark instances of the third set, IVNS improves the best known results (new lower bounds) in 28 cases and matches the best known results for the 55 remaining cases.

The rest of the paper is organized as follows. In the next Section, our IVNS algorithm and its components are described in detail. Section 3 is dedicated to computational assessments based on the commonly used benchmarks and comparisons with the state-of-the-art algorithms in the literature. In Section 4, several essential components of the proposed algorithm are investigated to shed light on how they affect the performance of the proposed algorithm. Concluding comments are summarized in the last section.

\section{Iterated Variable Neighborhood Search for the CCP}

Variable neighborhood search (VNS) [17,24] has been applied with success to many combinatorial optimization problems (see for instances $[1,5,25,31,32]$ ). In this work, we follow the general VNS framework and propose the iterated variable neighborhood search (IVNS) method for the CCP which integrates specially designed components to reach a suitable trade-off between intensification and diversification of the search process. Specifically, the proposed IVNS algorithm employs a randomized construction procedure to generate the initial solution, a new local optimization approach called the EVND method (extended variable neighborhood descent method) to discover local optima, and a shake procedure to perturb the incumbent solution. The proposed IVNS algorithm also employs a diversification stage to produce transition states between high-quality local optimum solutions. 


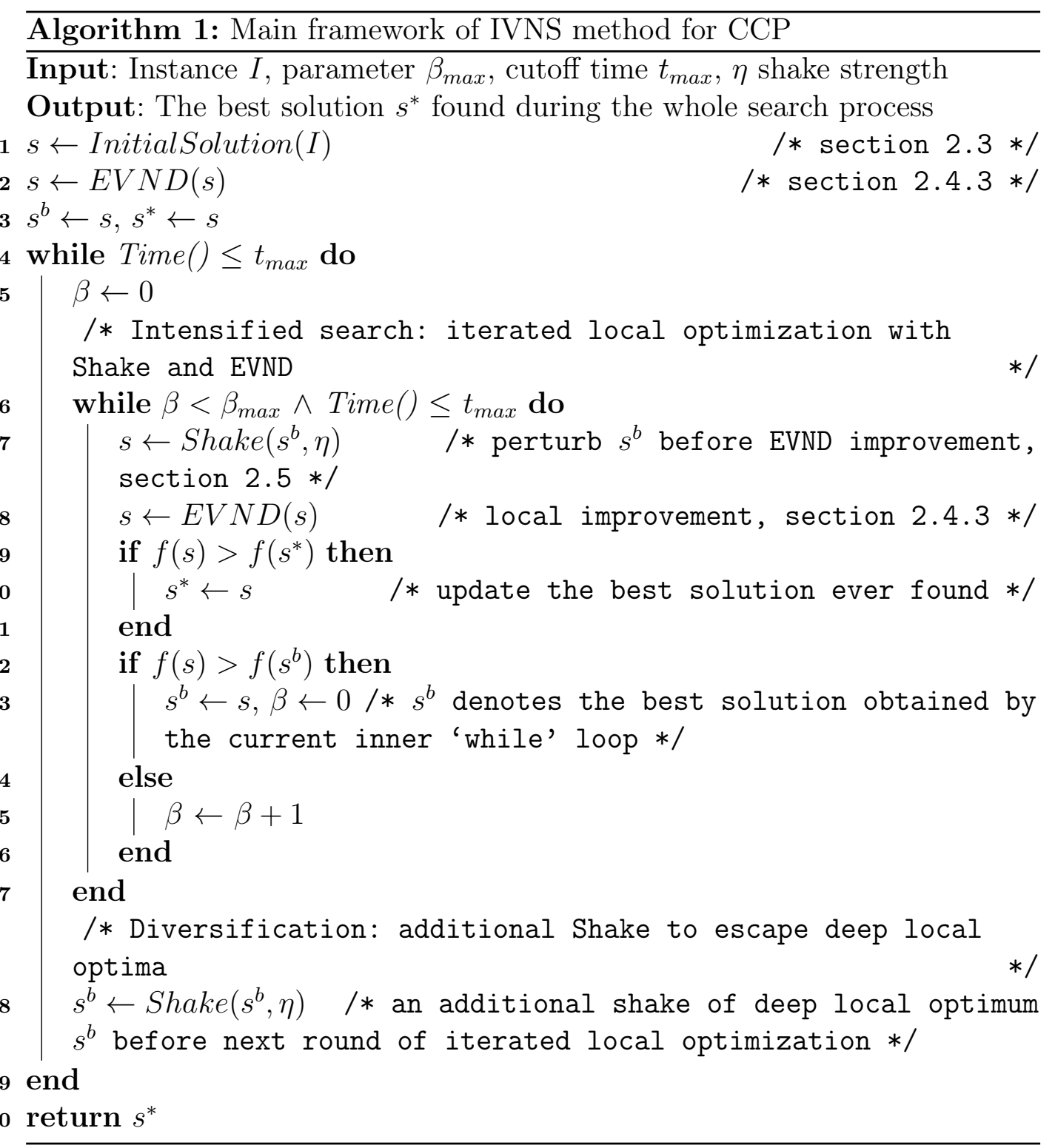

\subsection{General Procedure}

Our IVNS algorithm (Algorithm 1) starts from an initial feasible solution that is generated by a randomized construction procedure (Section 2.3) and is improved by the EVND method (lines 1 and 2, Section 2.4.3). Then it enters a 'while' loop in which an iterated local optimization (the inner 'while' loop, lines 5 to 17) and a diversification phase (the Shake call, line 18) are iteratively performed until a cutoff time $t_{\max }$ is reached.

The inner 'while' loop aims to find, from a given solution (a local optimum), an improved local optimum by iterating the Shake procedure (line 7) followed by the EVND procedure (line 8 ). The starting solution is first shaken by mak- 
ing $\eta$ changes ( $\eta$ is called shake strength, see Section 2.5) which serves as the starting point of the extended variable neighborhood descent procedure (see Section 2.4.3). The outcome of each EVND application is used to update the best solution ever found $\left(s^{*}\right.$, lines 9-11) and the best local optimum found during the current iterated local optimization phase $\left(s^{b}\right.$, lines 12-16). The counter $\beta$ indicates the number of consecutive local optimization (Shake+EVND) iterations during which $s^{b}$ is not updated ( $\beta$ is reset to 0 each time an improved local optimum $s^{b}$ is discovered). The inner 'while' loop exits when the cuttoff time is reached (in which case the whole algorithm terminates) or when $\beta$ attains a fixed value $\beta_{\max }$ (a large $\beta_{\max }$ thus induces a more intensified search). In the later case, $s^{b}$ indicates a deep local optimum that the inner Shake call (line 7) is not sufficient to help EVND to escape. For this reason, we apply an additional Shake call (line 18) to modify $s^{b}$ before giving it to the next round of the inner 'while' loop.

Note that with the second Shake call (line 18), the next inner 'while' loop starts the local optimization (EVND) with a doubly shaken starting solution, which diversifies the search strongly and helps escape deep local optima. More generally, the second Shake call may be replaced by other diversification techniques like random or customized restarts. In our case, we simply adopt the same Shake procedure used in the iterated local optimization phase. As shown in Section 3, this technique proves to be suitable and effective for the tested benchmarks. We also provide a study in Section 4.3 about the diversification effect of this second Shake application.

\subsection{Search Space, Evaluation Function and Solution Representation}

For a given CCP instance that is composed of a weighted graph $G=(V, E, C, w)$, the number $p$ of clusters, and the lower and upper limits $L$ and $U$ on the capacity of clusters, the search space $\Omega$ explored by the IVNS algorithm contains all feasible solutions, i.e., all partitions of the nodes of $V$ into $p$ clusters such that the weight of each cluster lies between its lower and upper limits.

Formally, let $\pi: V \rightarrow\{1, \ldots, p\}$ be a partition function of the $n$ nodes of $V$ to $p$ clusters. For each cluster $g \in\{1, \ldots, p\}$, let $\pi_{g}=\{v \in V: \pi(v)=g\}$ (i.e., $\pi_{g}$ is the set of nodes of cluster $g$ ). Then the search space $\Omega$ explored by our IVNS algorithm is given by:

$$
\Omega=\left\{\pi: \forall g \in\{1, \ldots, p\}, L \leq\left|\pi_{g}\right| \leq U,\left|\pi_{g}\right|=\sum_{v \in \pi_{g}} w(v)\right\}
$$

For any candidate partition $s=\left\{\pi_{1}, \pi_{2}, \ldots, \pi_{p}\right\}$ in $\Omega$, its quality is evaluated by the objective function value $f(s)$ of the CCP: 


$$
f(s)=\sum_{g=1}^{p} \sum_{v_{i}, v_{j} \in \pi_{g}, i<j} c_{i j}
$$

Given a candidate solution $s=\left\{\pi_{1}, \pi_{2}, \ldots, \pi_{p}\right\}$, IVNS employs a $n$-dimensional vector $x$ (element coordinate vector) to indicate the cluster of each node (or element). That is, if element $i$ belongs to cluster $\pi_{g}$, then $x[i]=g(i \in$ $\{1, \ldots, n\}$ ). IVNS additionally uses a $p$-dimensional vector $W C$ (cluster weight vector) to indicate the weight of each cluster of solution $s$, i.e., $W C[g]=$ $\sum_{v \in \pi_{g}} w(v)(\forall g \in\{1, \ldots, p\})$. Moreover, to facilitate neighborhood operations during the search process, the algorithm maintains a $n \times p$ matrix $\gamma$ in which the entry $\gamma[v][g]$ represents the sum of edge weights between the node $v$ and the nodes of cluster $g$ in the candidate solution $s$, i.e., $\gamma[v][g]=\sum_{u \in \pi_{g}} c_{v u}$. Consequently, any candidate solution $s \in \Omega$ can be conveniently indicated by the $x$ and $W C$ vectors, the $\gamma$ matrix and its objective function value $f$, i.e., $s$ $=<x, W C, \gamma, f>$.

\subsection{Initial Solution}

The proposed IVNS algorithm needs, for each run, an initial solution to start its search. In this work, we devise two randomized construction procedures for this purpose. The first procedure (Algorithm 2) operates in two stages. In the first stage, it iteratively performs a series of insertion operations until all clusters satisfy their lower capacity constraint. Specifically, for each insertion operation, a node $v$ and a cluster $g$ are randomly chosen from the set $A N$ of unassigned nodes and the set $A C$ of clusters whose lower bound constraint is not satisfied, then the node $v$ is assigned to cluster $g$. In the second stage, the construction procedure performs again a series of insertion operations until all nodes are assigned. Each insertion operation consists of randomly picking an unassigned node $v$ and a cluster $g$ such that $W C[g]+w(v) \leq U$, and then assigning $v$ to $g$, where $W C[g]$ and $w(v)$ denote respectively the current weight of cluster $g$ and the weight of node $v$.

However, the preliminary experiments showed that it was often difficult to obtain a feasible solution by the above procedure when the upper capacity limit of clusters is very tight. As a result, we modify slightly the above procedure as follows to obtain a second construction procedure. For each insertion operation, instead of randomly picking a node from the set $A N$ of unassigned nodes, we always choose the node $v$ in $A N$ such that $v$ has the largest weight (break ties at random).

Due to the random choices for insertion operations, the construction procedures are able to generate diversified initial solutions which allow the algorithm 


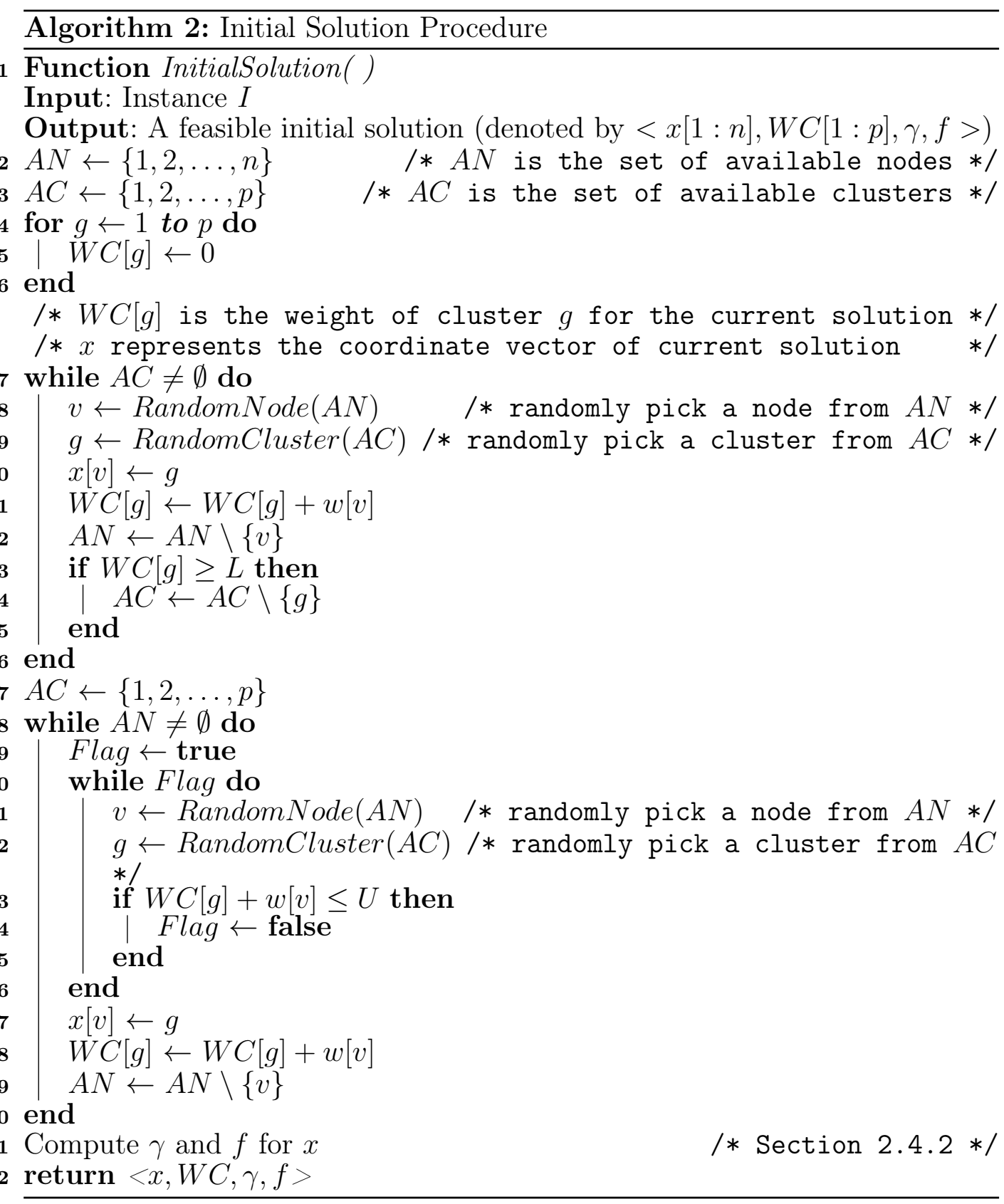

to start each run in a different area of the search space.

\subsection{Local Optimization Method}

Our IVNS algorithm employs the EVND method as its local optimization procedure which extends the standard variable neighborhood descent (VND) method. The detail of the EVND method is described in the following subsections. 
The neighborhood $N_{3}$ is based on the 2-1 exchange operator $(\operatorname{Exchange}(v, u, z))$. Given the current solution $s=\left\{\pi_{1}, \pi_{2}, \ldots, \pi_{p}\right\}$ and three nodes $v, u$ and $z$, where $v$ and $u$ are located in the same cluster $\pi_{i}$ and $z$ is located in another cluster $\pi_{j}$, Exchange $(v, u, z)$ transfers the nodes $v$ and $u$ from their current cluster $\pi_{i}$ to the cluster $\pi_{j}$, and transfers simultaneously the node $z$ from the cluster $\pi_{j}$ to the cluster $\pi_{i}$ in such a way that the resulting solution is still feasible. For the current solution $s$, the neighborhood $N_{3}$ of $s$ is composed of all feasible neighboring solutions which can be obtained by applying the 
Exchange $(v, u, z)$ operator to $s$ :

$$
\begin{array}{r}
N_{3}(s)=\left\{s \oplus \text { Exchange }(v, u, z): v, u \in \pi_{i}, z \in \pi_{j}, L \leq\left\{\left|\pi_{i}\right|-w(u)-w(v)+\right.\right. \\
\left.\left.w(z),\left|\pi_{j}\right|+w(v)+w(u)-w(z)\right\} \leq U, i \neq j\right\}
\end{array}
$$

Since the Exchange $(v, u, z)$ operator involves three nodes, the size of $N_{3}$ is bounded by $O\left(n^{3}\right)$ and is usually much larger than that of $N_{2}$ and $N_{1}$.

Additionally, it is worth noticing that these three neighborhoods (i.e., $N_{1}, N_{2}$, and $N_{3}$ ) are functionally complementary. Actually, the OneMove, SwapMove, and Exchange $(v, u, z)$ operators transfer at a time 1, 2, and 3 nodes, respectively. As a result, their combined use offers more opportunities for the local search procedure to disvover high-quality solutions.

\subsubsection{Fast Neighborhood Evaluation Technique}

Similar to the previous studies for the MDGP [5,21,28,29,31], our EVND procedure employs an incremental evaluation technique to calculate rapidly the move value (i.e., the change of objective value) of each candidate move. As mentioned in Section 2.2, our procedure maintains a $n \times p$ matrix $\gamma$ in which the entry $\gamma[v][g]$ represents the sum of weights between the node $v$ and the nodes of cluster $g$ for the current solution, i.e., $\gamma[v][g]=\sum_{u \in \pi_{g}} c_{v u}$. With the help of matrix $\gamma$, the evaluation function value $f$ can be calculated as $f(s)=$ $\frac{1}{2} \sum_{i=1}^{n} \gamma[i][x[i]]$ for an initial candidate solution $s=(x[1], x[2], \ldots, x[n])$. Moreover, the matrix $\gamma$ is frequently used in the neighborhood search operations (see Algorithms 4 to 6).

Based on the current solution (or partition) $s=\left\{\pi_{1}, \pi_{2}, \ldots, \pi_{p}\right\}$, if a OneMove operation $\left\langle v, \pi_{i}, \pi_{j}\right\rangle$ is performed, the move value can be easily determined as $\Delta_{f}\left(\left\langle v, \pi_{i}, \pi_{j}\right\rangle\right)=\gamma[v][j]-\gamma[v][i]$, and then the matrix $\gamma$ is accordingly updated. More specifically, the $i$-th and $j$-th columns of matrix $\gamma$ are updated as follows: $\gamma[u][i]=\gamma[u][i]-c_{v u}, \gamma[u][j]=\gamma[u][j]+c_{v u}, \forall u \in V, u \neq v$, where $c_{v u}$ is the edge weight between the nodes $v$ and $u$. As such, the evaluation function value $f$ can be rapidly updated as $f \leftarrow f+\Delta_{f}$.

When a $\operatorname{SwapMove}(v, u)$ operation is performed, its move value is calculated as $\Delta_{f}(\operatorname{SwapMove}(v, u))=(\gamma[v][x[u]]-\gamma[v][x[v]])+(\gamma[u][x[v]]-\gamma[u][x[u]])-$ $2 c_{v u}$, where $x[v]$ and $x[u]$ are the cluster of nodes $v$ and $u$ in the current solution $s$. As stated in Section 2.2, $x=(x[1], x[2], \ldots, x[n])$ represents the coordinate vector of the incumbent solution $s$. Since a SwapMove $(v, u)$ operation is composed of two consecutively performed OneMove operations, i.e., $s \oplus \operatorname{SwapMove}(v, u)=(s \oplus\langle v, x[v], x[u]\rangle) \oplus<u, x[u], x[v]\rangle$, matrix $\gamma$ is consecutively updated two times according to the OneMove move.

When a Exchange $(v, u, z)$ move is performed, the move value is calculated as 
$\Delta_{f}(\operatorname{Exchange}(v, u, z))=(\gamma[v][x[z]]-\gamma[v][x[v]])+(\gamma[u][x[z]]-\gamma[u][x[u]])+$ $(\gamma[z][x[v]]-\gamma[z][x[z]])+2\left(c_{v u}-c_{v z}-c_{u z}\right)$. Since a Exchange $(v, u, z)$ move is composed of three consecutively performed OneMove moves, i.e., $s \oplus$ Exchange $(v, u, z)=((s \oplus<v, x[v], x[z]>) \oplus\langle u, x[u], x[z]\rangle) \oplus\langle z, x[z], x[v]\rangle$, matrix $\gamma$ is consecutively updated three times according to the OneMove move.

Matrix $\gamma$ is initialized at the beginning of each run of the EVND procedure with a time complexity of $O\left(n^{2}\right)$, and is updated after each move operation in $O(n)$ for any considered move operator.

\subsubsection{Extended Variable Neighborhood Descent}

Algorithm 3: Extended Variable Neighborhood Descent (EVND) for CCP

1 Function $\operatorname{EVND}\left(s_{0}\right)$

Input: Solution $s_{0}$

Output: The local optimum solution $s$

$2 s \leftarrow s_{0}$

3 repeat

repeat

$s \leftarrow L S N_{1}(s)$

$($ Flag,$s) \leftarrow L S N_{2}(s)$

/* Algorithm $4 *$ /

until Flag = false

$($ Flag,$s) \leftarrow L S N_{3}(s)$

/* Algorithm $5 *$ /

until Flag = false

return $s$

Let $N_{k}\left(k=1,2, \ldots, k_{\max }\right)$ be a sequence of neighborhood structures (also called the neighborhood in this Section) with respect to a given optimization problem, the standard variable neighborhood descent (VND) method changes in a deterministic way the current neighborhood in order to find a highquality local optimum solution with respect to all $k_{\max }$ neighborhoods $[9,24]$. Specifically, the standard VND method starts with the first neighborhood $N_{1}$ $(k=1)$ and makes a complete exploitation of the neighborhood. Then, the VND method switches orderly to the next neighborhood $N_{k+1}(k \leftarrow k+1)$ when the current neighborhood $N_{k}\left(k=1,2, \ldots, k_{\max }-1\right)$ is fully explored without finding an improving solution. Moreover, the search process switches immediately to the first neighborhood $N_{1}$ as soon as an improving solution is detected in the current neighborhood $N_{k}$, i.e., $k \leftarrow 1$. Finally, the VND method stops if the search process reaches the last neighborhood $N_{k_{\max }}$ and no improving solution can be found in $N_{k_{\max }}$, and the best solution found during the search process is returned as the result of the VND method. Clearly, the returned solution is a local optimum solution with respect to all $k_{\max }$ neighborhoods. 


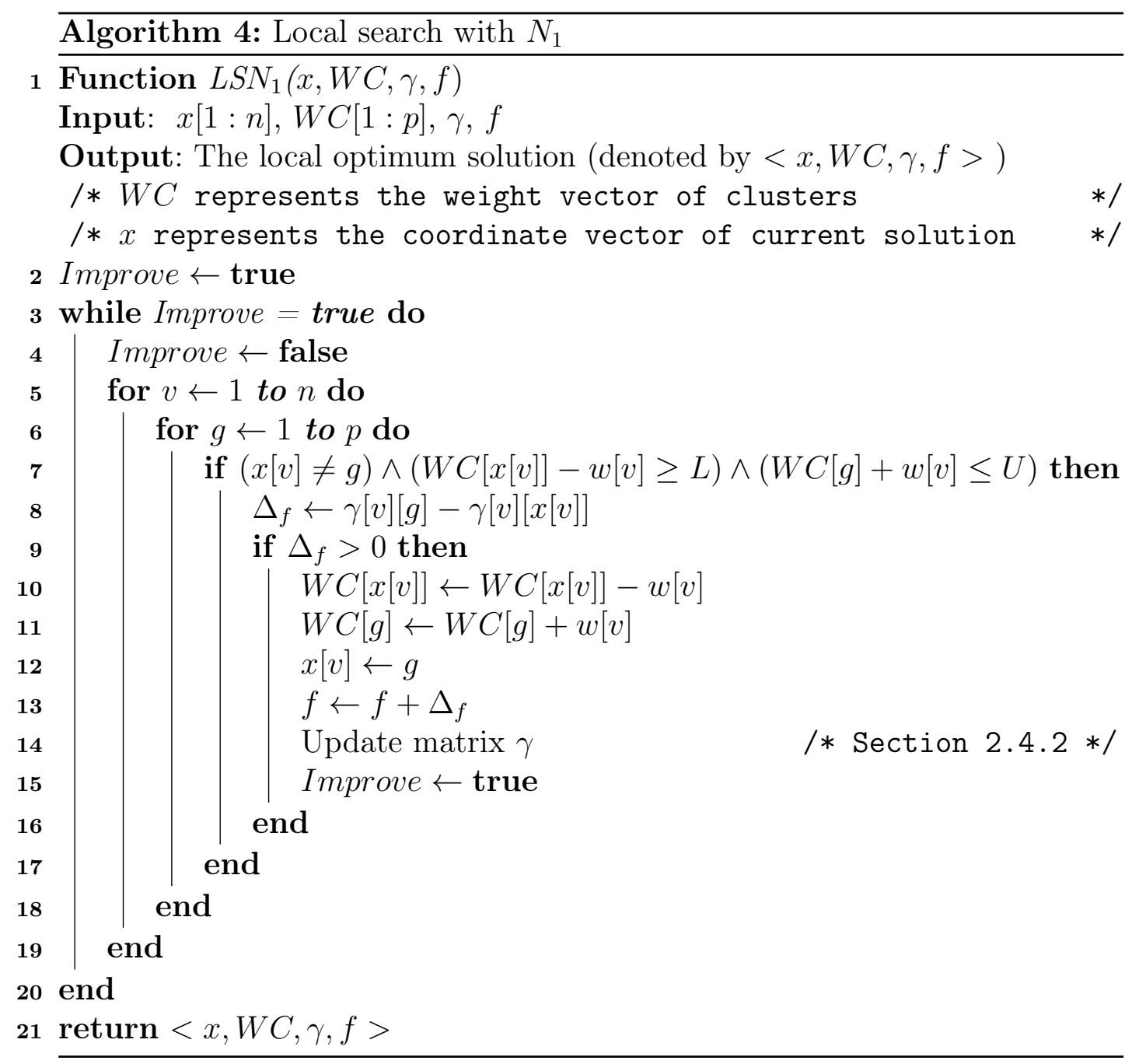

Our EVND method described in Algorithm 3 extends the standard VND method in the sense that EVND employs a different condition to switch from the current neighborhood $N_{k}(k>1)$ to the first neighborhood $N_{1}$. In the standard VND method, the search process switches back to the first neighborhood as soon as an improving solution is found in the current neighborhood $N_{k}$ (even if more improving solutions can be further found in $N_{k}$ ). However, our EVND method switches to the first neighborhood $N_{1}$ when one of the following two conditions is satisfied. First, the solution has been updated (or improved) $m$ ( $m \geq 1$, a parameter called 'the depth of improvement in neighborhood search') times with the current neighborhood $N_{k}$. Second, the solution has been updated (improved) at least one time with the neighborhood $N_{k}$ and no improving solution can further be found in the neighborhood $N_{k}$. Clearly, the standard VND method is a special case of our EVND method when $m=1$. Note that compared to the standard VND method, our EVND method imposes a stronger condition to move back to the first neighborhood.

Such an extension for the standard VND method is based on two consid- 


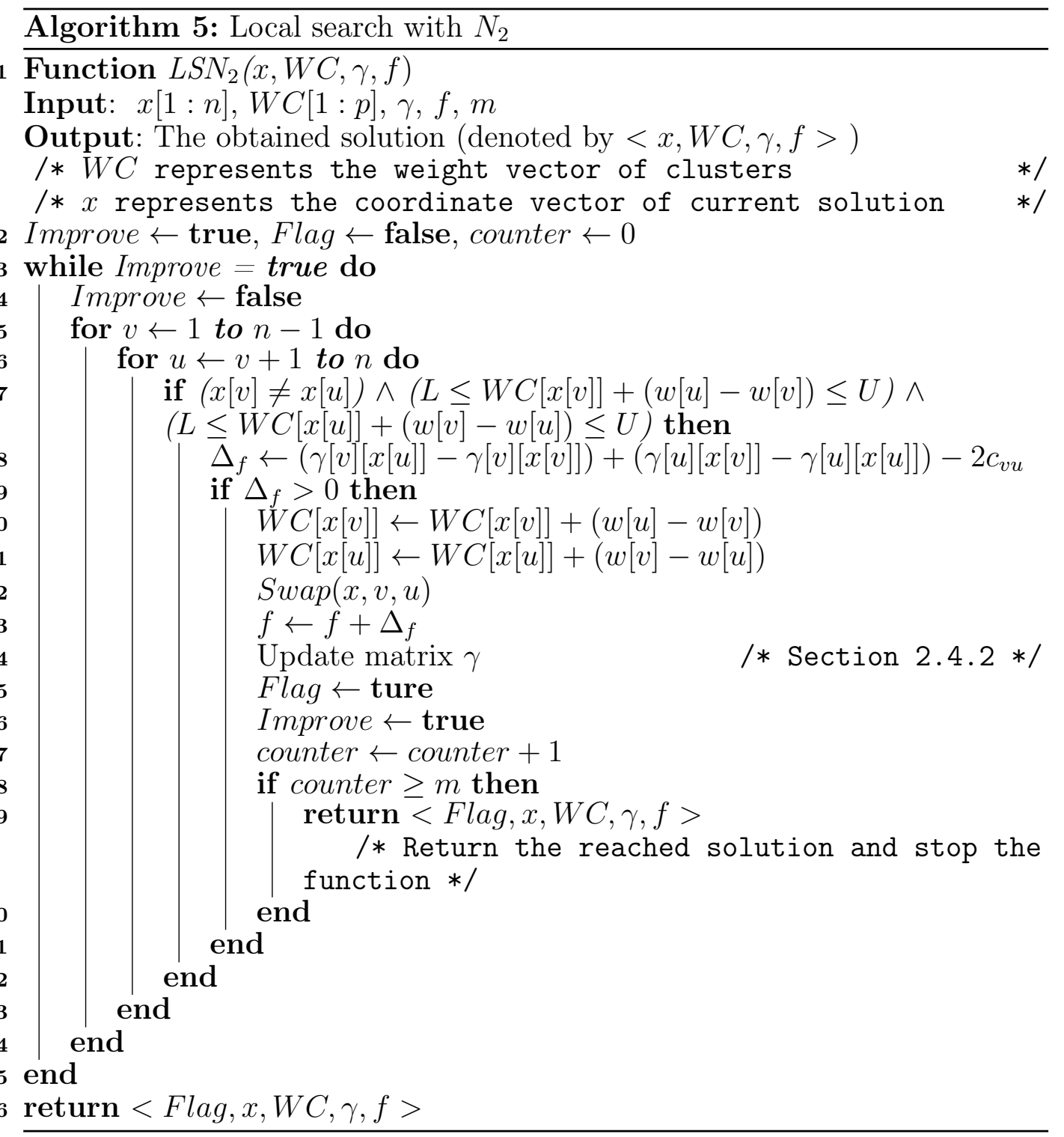

erations. First, in the standard VND method, the first neighborhood $N_{1}$ is explored more often than the other neighborhoods since we move back to $N_{1}$ as soon as an improving solution is discovered in the current neighborhood $N_{k}(k>1)$. However, a more balanced exploitation of all the $k$ neighborhoods constitutes another possibility and may help the search process to discover better solutions. Compared to the standard VND method, our EVND method promotes a more balanced exploitation of the neighborhoods $N_{k}\left(k=2,3, \ldots, k_{\max }\right)$ relative to the first neighborhood $N_{1}$. Second, the solutions returned by the neighborhoods $N_{k}\left(k=2,3, \ldots, k_{\max }\right)$ generally have a larger distance from the local optimum solution produced most recently by the first neighborhood $N_{1}$ with our EVND method than with the standard VND method. Thus, compared to the standard VND method, our EVND method creates some diversification effect during its intensified descent process with each neighborhood. 


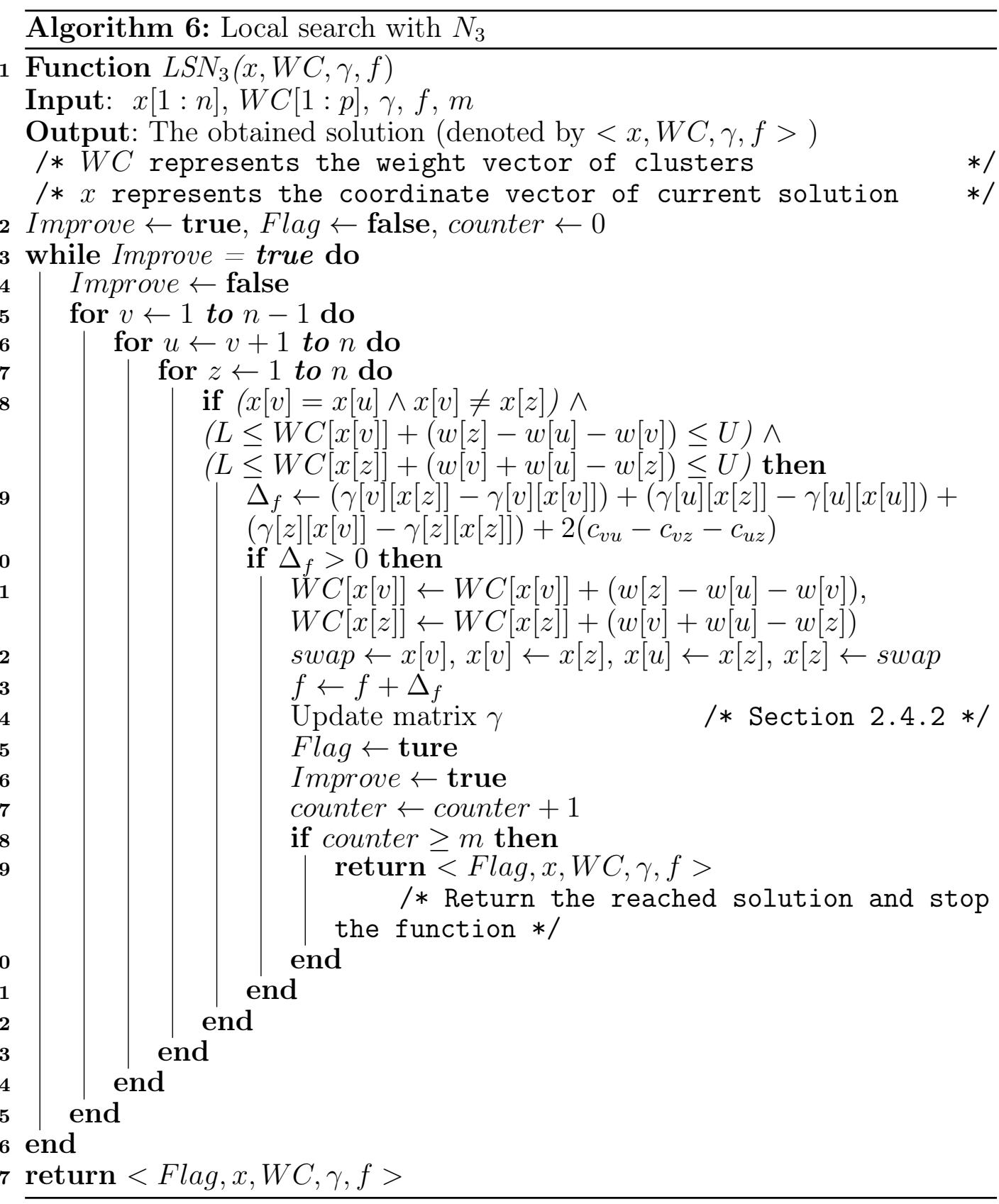

Our EVND method for the CCP exploits three complementary neighborhoods introduced in Section 2.4.1, i.e., $N_{1}, N_{2}$ and $N_{3}$ and is described in Algorithms 3 to 6 , where the variables $x, W C, \gamma$ and $f$ have the same meanings as those in Section 2.2.

From Algorithm 3, one can observe that our EVND procedure consists of two loops and each loop stops as long as the corresponding Flag variable receives the value false. Specifically, for the inner loop the Flag variable will get the value false when no improving neighbor exists in the neighborhood $N_{2}$ according to Algorithm 5. Similarly, for the outer loop the Flag variable will 


\subsection{Shake Procedure} incumbent solution.

get the value false when no improving neighbor exists in the neighborhood $N_{3}$ according to Algorithm 6. Consequently, the EVND procedure always stops when no improving neighbor exists in the neighborhoods $N_{2}$ and $N_{3}$ for the

Algorithm 7: Shake procedure

1 Function Shake $\left(s_{0}, \eta\right)$

Input: Solution $s_{0}$, strength of shake $\eta$

Output: The perturbed solution $s$, matrix $\gamma$, objective value $f$

$2 \leftarrow s_{0}$

3 for $l \leftarrow 1$ to $\eta$ do

$4 \quad$ Randomly pick a neighboring solution $s^{\prime} \in N_{1}(s) \cup N_{2}(s)$

$5 \quad s \leftarrow s^{\prime}$

6 end

7 Compute $\gamma$ and $f$ for $s$

8 return $\langle s, \gamma, f\rangle$

When our IVNS algorithm reaches a local optimum solution, we apply a Shake procedure to the reached solution to jump out of the local optimum trap. The Shake procedure used by the IVNS algorithm consists of consecutively performing $\eta$ randomly selected feasible OneMove or SwapMove moves, where $\eta$ is a parameter called the shake strength. In other words, from the incumbent solution $s_{0}$, we construct the $N_{1}(s)$ and $N_{2}(s)$ neighborhoods which include all (feasible) neighboring solutions of $s$ (see Section 2.4.1) and then pick randomly a solution $s^{\prime}$ from the union of $N_{1}(s)$ and $N_{2}(s)$ to replace $s_{0}$. We repeat this operation $\eta$ times. It is clear that a large (respect. small) $\eta$ value leads to a shaken solution which will be more (respect. less) distant from the input solution. In this work, the value of $\eta$ is empirically set as $\eta=\min \{15, \max \{5,0.02 n\}\}$, where $n$ is the number of nodes in the graph. Note that it is possible to include Exchange moves for the Shake operations. Meanwhile, for the reason of simplicity, we only apply OneMove and SwapMove moves, which proves to be sufficient for our purpose of diversification. The pseudo-code of our Shake procedure is given in Algorithm 7.

\section{Experimental Results and Comparisons}

In this section, we assess the performance of the proposed IVNS algorithm by showing computational results on well-known benchmark instances and by making a comparison with the state-of-the-art algorithms in the literature. 
Our IVNS algorithm was assessed on three sets of 133 benchmark instances commonly used in the literature. These instances are available at http:// www.optsicom.es/ccp/, and their details are described as follows.

- RanReal Set (40 instances): This set was originally proposed in [14] for the MDGP and adapted to the CCP in [23] by generating the node weights with a uniform distribution $\mathrm{U}(0,10)$. This set is composed of 20 instances with $n=240, p=12, L=75$, and $U=125$, and 20 instances with $n=480$, $p=20, L=100$, and $U=150$. For all instances of this set, the edge weights $c_{i j}$ are a real number which is uniformly and randomly generated in $(0,100)$.

- DB Set (10 instances): This set was originally proposed by Deng and Bard [9] for the MDGP in the context of mail delivery, and adapted to the CCP in [23] by generating the node weights with a uniform distribution $\mathrm{U}(0,10)$. These 10 instances are characterized by the following features: $n=82$, $p=8, L=25$, and $U=75$.

- MM Set (83 instances): These 83 synthetic instances were proposed by Morán-Mirabal et al. [26] for the handover minimization problem and were widely used in the literature. These instances have the following characteristics: $n \in\{20,30,40,100,200,400\}, p \in\{5,10,15,25,50\}$, the edge weights $c_{i j}$ are a real number, and the lower and upper capacity limits of clusters respectively are 0 and a real number depending on each instance.

\subsection{Parameter Settings and Experimental Protocol}

Table 1

Settings of parameters

\begin{tabular}{llll}
\hline Parameters & Section & Description & Values \\
\hline$\beta_{\max }$ & 2.1 & strength of intensification search & 30 \\
$m$ & 2.4 .3 & depth of improvement in neighborhood search & 10 \\
$\eta$ & 2.5 & strength of shake & $\min \{15, \max \{5,0.02 n\}\}$ \\
\hline
\end{tabular}

In this Section, we show some basic information about our experiments, including the parameter settings of our algorithm, the reference algorithms, the experimental platform, and the termination criterion of algorithms.

First, Table 1 shows the parameter setting of our IVNS algorithm which was achieved by a preliminary experiment. For this preliminary experiment, we used 20 RanReal instances with $n=240$ which were also used in the sensibility analysis of parameters presented in Section 4.4. The computational results indicated that for $m$ and $\beta_{\max }$ the default settings shown in Table 1 are suitable for the algorithm (see Section 4.4). For $\eta$, it involves three variables, so we manually tuned its value based on a principle that the strength of 
1 The source codes of these algorithms will be available at: http://www.info. univ-angers.fr/pub/hao/ccp.html

2 dmclique, ftp://dimacs.rutgers.edu/pub/dsj/clique

shake procedure should be proportional to the size of instance but in an appropriate interval. The computational results on the preliminary experiment indicated that the default setting of $\eta$ in Table 1 is able to reach an acceptable performance of the algorithm.

Second, according to the previous surveys [23,26], the TS [23], GRASP+TS [23], and GevPR-HMP [26] algorithms can be considered as the state-of-theart algorithms for the CCP. Hence, in the present study we use them as the main reference algorithms for our comparative study.

Our IVNS algorithm was programmed in $\mathrm{C}++$. To make a fair comparison with the state-of-the-art algorithms, we also implemented faithfully the GRASP, TS, GRASP + TS algorithms of [23] which are three state-of-the-art algorithms in the literature ${ }^{1}$. For the GRASP, TS, GRASP + TS algorithms, we adopted the best parameter settings identified in the original paper [23]. Moreover, all source codes were compiled using $\mathrm{g}++$ compiler with the '-O3' flag, and the corresponding experiments were carried out on a computing platform with an Intel E5-2670 processor (2.80 GHz CPU and 2Gb RAM), running Linux. Following the DIMACS machine benchmark procedure, our machine requires respectively $0.19,1.17$, and 4.54 seconds for the graphs r300.5, r400.5, $\mathrm{r} 500.5^{2}$.

For the GRASP, TS, GRASP+TS, and IVNS algorithms, we used a cutoff time $t_{\max }=n$ (in seconds) as the unique stopping criterion where $n$ is the number of nodes of the input graph.

Finally, for the IVNS algorithm, the initial solution was generated by the second initialization procedure for the handover minimization instances due to their tight upper bounds on the capacity of clusters, and by Algorithm 2 for the remaining instances. For GRASP, TS and GRASP + TS, the handover minimization instances are not used in the experiments, since their initial solution procedures can not guarantee to generate a feasible solution for a part of them due to the tight upper bounds on the capacity of clusters.

\subsection{Computational Results and Comparison on the general CCP Instances}

The first experiment aims to assess the performance of our IVNS algorithm on the first two sets of instances by comparing its results with those of the stateof-the-art algorithms in the literature. In this experiment, all the compared algorithms (GRASP, TS, GRASP + TS and IVNS) were respectively performed 
Table 2

Comparison between the IVNS algorithm and three state-of-the-art algorithms from the literature (i.e., GRASP, TS, GRASP + TS in [25]) on the first two sets (RanReal and $\mathrm{DB}$ ) of CCP instances in terms of the best and average objective function values over 20 independent runs. The best results among the compared algorithms are indicated in bold.

\begin{tabular}{|c|c|c|c|c|c|c|c|c|}
\hline \multirow[b]{2}{*}{ Instance } & \multicolumn{4}{|c|}{$f_{\text {best }}$} & \multicolumn{4}{|c|}{$f_{a v g}$} \\
\hline & GRASP & $\mathrm{TS}$ & GRASP+TS & IVNS & GRASP & $\mathrm{TS}$ & GRSP+TS & IVNS \\
\hline Sparse82_01 & 1342.17 & 1336.82 & 1342.17 & 1342.17 & 1342.13 & 1315.21 & 1342.17 & 1342.17 \\
\hline Sparse82_02 & 1306.64 & 1303.17 & 1306.64 & 1306.64 & 1305.65 & 1281.57 & 1306.16 & 1306.64 \\
\hline Sparse82_03 & 1353.94 & 1353.94 & 1353.94 & 1353.94 & 1351.69 & 1335.89 & 1353.00 & 1353.94 \\
\hline Sparse82_04 & 1291.22 & 1291.22 & 1291.22 & 1291.22 & 1289.15 & 1276.85 & 1290.05 & 1291.22 \\
\hline Sparse82_05 & 1352.35 & 1352.35 & 1352.35 & 1352.35 & 1352.35 & 1328.15 & 1352.35 & 1352.35 \\
\hline Sparse82_06 & 1354.61 & 1354.61 & 1354.61 & 1354.61 & 1353.86 & 1329.86 & 1354.61 & 1354.61 \\
\hline Sparse82_07 & 1266.94 & 1266.94 & 1266.94 & 1266.94 & 1266.86 & 1227.01 & 1266.89 & 1266.94 \\
\hline Sparse82_08 & 1393.02 & 1391.53 & 1393.02 & 1393.02 & 1393.02 & 1362.54 & 1393.02 & 1393.02 \\
\hline Sparse82_09 & 1294.12 & 1294.12 & 1294.12 & 1294.12 & 1293.69 & 1280.97 & 1293.46 & 1294.12 \\
\hline Sparse82_10 & 1356.98 & 1356.98 & 1356.98 & 1356.98 & 1356.85 & 1330.79 & 1356.95 & 1356.98 \\
\hline RanReal240_01 & 192320.30 & 222871.98 & 223272.87 & 224893.92 & 191140.45 & 221547.29 & 222268.07 & 224785.27 \\
\hline RanReal240_02 & 185612.48 & 202356.36 & 202344.44 & 204608.66 & 183435.92 & 200582.86 & 200356.15 & 204415.88 \\
\hline RanReal240_03 & 179316.65 & 196422.35 & 196143.12 & 198885.19 & 176821.81 & 194348.91 & 194783.74 & 198626.93 \\
\hline RanReal240_04 & 197342.26 & 222298.86 & 223076.30 & 225627.16 & 194629.26 & 220521.18 & 221165.75 & 225227.11 \\
\hline RanReal240_05 & 175967.25 & 193358.53 & 194115.62 & 195440.94 & 174465.68 & 191234.34 & 192076.34 & 195228.86 \\
\hline RanReal240_06 & 192789.55 & 214840.96 & 215004.12 & 216736.00 & 188264.62 & 212626.52 & 213259.64 & 216474.84 \\
\hline RanReal240_07 & 191714.87 & 208223.05 & 208045.67 & 209273.70 & 190379.19 & 205808.95 & 206092.48 & 209004.05 \\
\hline RanReal240_08 & 185930.72 & 203595.13 & 203168.62 & 205246.82 & 181699.18 & 201102.55 & 201519.15 & 204958.19 \\
\hline RanReal240_09 & 189573.48 & 207711.19 & 207984.26 & 209059.28 & 186992.97 & 206540.74 & 206788.13 & 208789.79 \\
\hline RanReal240_10 & 176327.61 & 189597.87 & 190532.75 & 192977.28 & 174638.77 & 187534.34 & 188379.13 & 192788.59 \\
\hline RanReal240_11 & 184198.31 & 203109.91 & 203037.25 & 204722.75 & 182673.54 & 201113.86 & 201701.83 & 204523.95 \\
\hline RanReal240_12 & 181337.55 & 199710.82 & 199708.68 & 201052.53 & 180048.84 & 198365.85 & 198389.30 & 200904.16 \\
\hline RanReal240_13 & 180865.29 & 201238.18 & 200742.90 & 202335.99 & 179893.27 & 199590.02 & 198727.63 & 202139.55 \\
\hline RanReal240_14 & 194009.94 & 226813.94 & 226621.92 & 228844.44 & 191947.27 & 225362.61 & 225721.97 & 228512.11 \\
\hline RanReal240_15 & 173114.22 & 188896.07 & 188318.39 & 191170.98 & 171311.83 & 187410.50 & 187299.92 & 190914.31 \\
\hline RanReal240_16 & 182348.50 & 202475.44 & 202463.27 & 203999.02 & 180823.13 & 199999.63 & 200722.29 & 203834.68 \\
\hline RanReal240_17 & 181270.70 & 194155.13 & 193835.64 & 195242.31 & 179607.16 & 191978.36 & 191908.95 & 195114.49 \\
\hline RanReal240_18 & 174650.37 & 192772.21 & 193004.84 & 195069.62 & 173876.39 & 190428.51 & 190979.42 & 194853.70 \\
\hline RanReal240_19 & 179859.43 & 196739.04 & 196717.17 & 199200.03 & 177521.56 & 194916.62 & 195053.62 & 199019.23 \\
\hline RanReal240_20 & 191936.64 & 210399.94 & 210365.38 & 212264.10 & 190110.07 & 208970.68 & 208887.59 & 212046.92 \\
\hline RanReal480_01 & 463538.35 & 543259.55 & 544301.19 & 555057.10 & 460340.75 & 539074.11 & 539550.62 & 554331.89 \\
\hline RanReal480_02 & 446829.64 & 500646.59 & 502039.41 & 510418.44 & 443573.33 & 495273.27 & 497700.18 & 509519.84 \\
\hline RanReal480_03 & 434854.27 & 486379.91 & 487561.06 & 496641.22 & 433059.42 & 482624.38 & 483908.86 & 495847.80 \\
\hline RanReal480_04 & 455470.88 & 510971.67 & 513425.37 & 521984.68 & 450861.04 & 504054.37 & 507123.99 & 520891.75 \\
\hline RanReal480_05 & 415295.30 & 474548.74 & 473732.77 & 483228.99 & 413263.81 & 466932.68 & 469914.28 & 482595.19 \\
\hline RanReal480_06 & 461624.20 & 524191.64 & 524520.14 & 533762.18 & 458049.90 & 519002.55 & 520607.74 & 532888.64 \\
\hline RanReal480_07 & 461236.11 & 537464.01 & 537674.45 & 545157.68 & 456319.85 & 530513.86 & 532199.71 & 544530.14 \\
\hline RanReal480_08 & 460756.67 & 521894.07 & 523602.31 & 532308.09 & 458299.68 & 515438.23 & 518685.30 & 531417.94 \\
\hline RanReal480_09 & 466977.73 & 546057.32 & 546394.74 & 556478.39 & 462121.78 & 540459.20 & 541615.49 & 555098.72 \\
\hline RanReal480_10 & 447088.04 & 508294.13 & 508168.74 & 519456.96 & 441810.74 & 503161.12 & 504750.89 & 518612.02 \\
\hline RanReal480_11 & 451321.35 & 515296.61 & 515189.66 & 523450.04 & 448295.75 & 510410.05 & 509657.31 & 522814.96 \\
\hline RanReal480_12 & 434343.91 & 492469.23 & 493845.25 & 501596.63 & 433067.26 & 487442.12 & 488699.18 & 500580.84 \\
\hline RanReal480_13 & 467130.54 & 526936.22 & 524825.92 & 534638.19 & 461557.62 & 519201.37 & 521740.67 & 533763.20 \\
\hline RanReal480_14 & 428544.75 & 500100.04 & 508349.48 & 513777.84 & 426455.75 & 495859.32 & 499543.12 & 512975.73 \\
\hline RanReal480_15 & 446764.19 & 509377.07 & 509005.17 & 516941.11 & 443810.07 & 503159.99 & 503946.63 & 516017.98 \\
\hline RanReal480_16 & 465499.89 & 540493.75 & 540840.67 & 549371.23 & 462345.11 & 533502.50 & 535129.37 & 548276.15 \\
\hline RanReal480_17 & 460122.80 & 531353.71 & 529388.54 & 537483.76 & 458004.02 & 523459.84 & 524362.15 & 536655.06 \\
\hline RanReal480_18 & 456573.73 & 515692.20 & 518675.12 & 525813.39 & 452767.79 & 511193.51 & 512917.14 & 524650.86 \\
\hline RanReal480_19 & 454922.45 & 514503.74 & 512339.77 & 522158.86 & 449744.91 & 506858.76 & 508311.67 & 521180.84 \\
\hline RanReal480_20 & 443851.41 & 510045.22 & 509167.33 & 518288.03 & 440398.01 & 503995.42 & 505190.58 & 517261.92 \\
\hline \#Best & 10 & 7 & 10 & 50 & 2 & 0 & 4 & 50 \\
\hline$p$-value & $2.54 \mathrm{e}-10$ & $5.47 \mathrm{e}-11$ & $2.54 \mathrm{e}-10$ & & $4.26 \mathrm{e}-12$ & $1.54 \mathrm{e}-12$ & $1.18 \mathrm{e}-11$ & \\
\hline
\end{tabular}


Table 3

Comparison between the IVNS algorithm and three state-of-the-art algorithms from the literature (i.e., GRASP, TS, GRASP+TS [25]) on the first two sets (RanReal and $\mathrm{DB}$ ) of CCP instances in terms of the standard deviation and the average running time to reach its final objective value. Each instance was independently solved 20 times by each algorithm respectively.

\begin{tabular}{|c|c|c|c|c|c|c|c|c|}
\hline \multirow[b]{2}{*}{ Instance } & \multicolumn{4}{|c|}{ standard deviation $(\sigma)$} & \multicolumn{4}{|c|}{ time avg $(s)$} \\
\hline & GRASP & TS & GRASP+TS & IVNS & GRASP & TS & GRASP+TS & IVNS \\
\hline Sparse82_01 & 0.16 & 23.24 & 0.00 & 0.00 & 14.01 & 9.31 & 15.15 & 0.13 \\
\hline Sparse82_02 & 0.93 & 19.99 & 0.97 & 0.00 & 30.82 & 7.53 & 29.27 & 0.82 \\
\hline Sparse82_03 & 1.24 & 14.90 & 1.63 & 0.00 & 41.60 & 2.38 & 38.78 & 0.21 \\
\hline Sparse82_04 & 1.32 & 12.97 & 1.04 & 0.00 & 30.60 & 6.36 & 36.99 & 4.27 \\
\hline Sparse82_05 & 0.00 & 30.33 & 0.00 & 0.00 & 4.97 & 8.02 & 5.11 & 0.07 \\
\hline Sparse82_06 & 1.39 & 25.63 & 0.00 & 0.00 & 24.43 & 4.03 & 26.04 & 0.08 \\
\hline Sparse82_07 & 0.18 & 22.46 & 0.10 & 0.00 & 32.74 & 4.75 & 28.10 & 0.39 \\
\hline Sparse82_08 & 0.00 & 29.24 & 0.00 & 0.00 & 1.00 & 9.31 & 0.74 & 0.03 \\
\hline Sparse82_09 & 0.40 & 13.01 & 0.22 & 0.00 & 28.54 & 5.67 & 16.31 & 0.58 \\
\hline Sparse82_10 & 0.16 & 24.48 & 0.07 & 0.00 & 29.41 & 2.08 & 37.81 & 0.59 \\
\hline RanReal240_01 & 864.05 & 1098.76 & 601.21 & 97.88 & 166.10 & 15.24 & 132.87 & 147.28 \\
\hline RanReal240_02 & 936.74 & 1140.64 & 1886.92 & 102.77 & 128.76 & 17.81 & 132.97 & 160.27 \\
\hline RanReal240_03 & 786.12 & 1192.60 & 875.04 & 170.83 & 123.40 & 7.19 & 125.43 & 146.88 \\
\hline RanReal240_04 & 1018.21 & 1302.30 & 1060.61 & 237.09 & 122.63 & 13.49 & 146.27 & 162.63 \\
\hline RanReal240_05 & 646.56 & 1255.73 & 894.36 & 91.62 & 113.10 & 8.38 & 129.66 & 161.77 \\
\hline RanReal240_06 & 1321.33 & 1251.75 & 959.32 & 169.61 & 128.25 & 15.04 & 139.80 & 165.88 \\
\hline RanReal240_07 & 592.59 & 1696.59 & 1324.09 & 120.82 & 114.48 & 27.88 & 143.93 & 120.41 \\
\hline RanReal240_08 & 1271.82 & 1184.11 & 1096.61 & 139.71 & 137.49 & 19.52 & 128.97 & 174.29 \\
\hline RanReal240_09 & 1127.57 & 1073.39 & 873.02 & 148.77 & 135.58 & 27.24 & 158.97 & 160.17 \\
\hline RanReal240_10 & 659.31 & 1160.27 & 1080.52 & 154.18 & 107.53 & 7.40 & 127.40 & 170.78 \\
\hline RanReal240_11 & 855.28 & 1127.48 & 1041.35 & 96.57 & 115.17 & 36.41 & 162.40 & 148.56 \\
\hline RanReal240_12 & 624.13 & 1347.08 & 961.73 & 126.79 & 128.65 & 34.82 & 151.19 & 169.33 \\
\hline RanReal240_13 & 531.24 & 1095.26 & 1191.67 & 155.41 & 102.67 & 38.44 & 131.83 & 150.75 \\
\hline RanReal240_14 & 1185.33 & 1086.76 & 672.46 & 170.71 & 112.17 & 10.11 & 124.59 & 141.27 \\
\hline RanReal240_15 & 780.67 & 1026.16 & 700.91 & 174.08 & 120.50 & 8.78 & 126.35 & 144.95 \\
\hline RanReal240_16 & 645.97 & 1341.25 & 1033.99 & 131.32 & 97.79 & 19.38 & 141.58 & 147.86 \\
\hline RanReal240_17 & 679.79 & 1280.03 & 936.77 & 109.08 & 120.85 & 25.01 & 142.44 & 146.03 \\
\hline RanReal240_18 & 458.00 & 1155.66 & 1050.83 & 126.17 & 141.20 & 7.55 & 130.71 & 163.49 \\
\hline RanReal240_19 & 924.99 & 1173.30 & 1357.67 & 109.79 & 113.09 & 8.71 & 125.27 & 176.31 \\
\hline RanReal240_20 & 829.60 & 908.52 & 816.33 & 130.92 & 99.00 & 24.49 & 146.64 & 149.66 \\
\hline RanReal480_01 & 1767.54 & 2915.75 & 2305.94 & 415.49 & 276.78 & 87.81 & 270.05 & 369.26 \\
\hline RanReal480_02 & 1541.69 & 3027.29 & 1982.60 & 569.71 & 212.88 & 50.36 & 276.25 & 416.40 \\
\hline RanReal480_03 & 909.49 & 2529.01 & 1999.88 & 426.86 & 281.57 & 55.11 & 274.68 & 415.80 \\
\hline RanReal480_04 & 1426.35 & 4323.08 & 2867.38 & 648.97 & 230.07 & 53.23 & 275.02 & 380.20 \\
\hline RanReal480_05 & 1094.52 & 3383.90 & 1886.34 & 497.25 & 310.87 & 33.56 & 271.51 & 338.96 \\
\hline RanReal480_06 & 1573.22 & 3178.92 & 2393.40 & 555.89 & 205.83 & 58.20 & 291.32 & 338.25 \\
\hline RanReal480_07 & 1926.89 & 2864.48 & 2451.06 & 413.13 & 198.46 & 63.44 & 291.77 & 388.74 \\
\hline RanReal480_08 & 1404.82 & 4291.62 & 2058.61 & 555.42 & 194.59 & 77.79 & 291.08 & 373.48 \\
\hline RanReal480_09 & 1994.95 & 3774.05 & 2163.84 & 514.64 & 231.09 & 78.00 & 282.38 & 388.08 \\
\hline RanReal480_10 & 1667.97 & 2799.90 & 2715.16 & 589.69 & 296.32 & 49.75 & 268.99 & 403.46 \\
\hline RanReal480_11 & 1488.69 & 2771.21 & 2220.12 & 402.37 & 243.62 & 98.13 & 277.81 & 382.52 \\
\hline RanReal480_12 & 1078.08 & 3170.40 & 2713.09 & 540.16 & 292.27 & 80.68 & 296.84 & 386.04 \\
\hline RanReal480_13 & 2379.98 & 3611.97 & 2431.74 & 423.89 & 264.67 & 66.61 & 289.75 & 420.59 \\
\hline RanReal480_14 & 1025.07 & 2482.93 & 2984.86 & 408.22 & 275.91 & 41.00 & 276.64 & 401.09 \\
\hline RanReal480_15 & 1358.30 & 3001.21 & 3149.15 & 408.07 & 241.74 & 86.60 & 296.67 & 375.96 \\
\hline RanReal480_16 & 1331.41 & 4488.81 & 2636.34 & 590.34 & 217.53 & 56.34 & 279.50 & 366.12 \\
\hline RanReal480_17 & 1306.58 & 3384.98 & 2538.33 & 402.38 & 274.19 & 56.09 & 286.52 & 389.54 \\
\hline RanReal480_18 & 1343.73 & 4530.39 & 2388.17 & 494.86 & 248.92 & 80.52 & 307.61 & 379.04 \\
\hline RanReal480_19 & 2115.36 & 2949.64 & 2357.29 & 550.77 & 248.76 & 49.19 & 282.87 & 405.61 \\
\hline RanReal480_20 & 1146.60 & 3505.30 & 2635.41 & 530.58 & 255.89 & 91.93 & 288.15 & 398.10 \\
\hline \#Best & 2 & 0 & 4 & 50 & & & & \\
\hline$p$-value & $4.26 \mathrm{e}-12$ & $1.54 \mathrm{e}-12$ & $1.18 \mathrm{e}-11$ & & & & & \\
\hline
\end{tabular}


20 times on each instance, based on the experimental protocol of Section 3.2. The computational results are summarized in Tables 2 and 3.

In Table 2 , the first column identifies the instances, columns 2-4 show respectively the best objective values $\left(f_{\text {best }}\right)$ obtained by the three reference algorithms (GRASP, TS, GRASP+TS), and column 5 reports the best objective values of our IVNS algorithm. Columns 6-9 show respectively the average objective values for the four compared algorithms $\left(f_{\text {avg }}\right)$. The best results among the algorithms in terms of the best and average objective values are indicated in bold. In Table 3, columns 2-5 show the standard deviation $(\sigma)$ of the objective values obtained over 20 runs for the compared algorithms, respectively, and columns 6-9 give the average running times (in seconds) of the algorithms to reach their respective objective values $\left(\right.$ time $\left._{\text {avg }}\right)$. The row \#Best of the tables indicates the number of instances for which the corresponding algorithm produces the best results among the compared algorithms. In addition, to verify whether there exists a significant difference between the reference algorithms and our IVNS algorithm on the best and average objective values, as well as the standard deviation of objective values, the $p$-values from the non-parametric Friedman test are reported in the last row of the tables. Notice that a $p$-value smaller than 0.05 means that there exists a significant difference between two sets of results compared.

One observes from Tables 2 and 3 that the proposed IVNS algorithm outperforms the reference algorithms. First, IVNS obtained the best result on all 50 instances in terms of the best objective value, whereas the GRASP, TS, GRASP+TS algorithms produced respectively the best result on 10, 7 and 10 instances. Second, when comparing the average objective values, it can be found that the IVNS algorithm yielded the best result on all instances, whereas the GRASP, TS, and GRASP+TS algorithms respectively obtained the best results on only $2,0,4$ instances. In addition, the small $p$-values $(<0.05)$ confirm the significant differences between the results of IVNS and those of the compared reference algorithms.

Finally, compared to the reference algorithms, the IVNS algorithm produced the smallest standard deviation $(\sigma)$ on all tested instances, indicating that IVNS is the most robust algorithm among the compared algorithms, which is also confirmed by the associated small $p$-values.

\subsection{Computational Results and Comparison on the Handover Minimization Instances}

The second experiment aims to assess the performance of the IVNS algorithm on the set of 83 handover minimization instances with $n \leq 400$, where for each 
Table 4

Comparison between the IVNS algorithm and the three reference algorithms in [26] on the set of handover minimization instances. Each instance was independently solved 20 times by the IVNS algorithm, and the current best results are indicated in bold. The results are given in the form of minimization to make a direct comparison with the results from the literature.

\begin{tabular}{|c|c|c|c|c|c|c|c|c|c|}
\hline \multirow[t]{2}{*}{ Instance } & \multirow[t]{2}{*}{ BKS } & \multirow{2}{*}{$\begin{array}{l}\text { GevPR- } \\
\text { HMP } \\
f_{\text {best }}\end{array}$} & \multirow{2}{*}{$\begin{array}{l}\text { GQAP } \\
f_{\text {best }}\end{array}$} & \multirow{2}{*}{$\begin{array}{l}\text { BRKGA } \\
f_{\text {best }}\end{array}$} & \multicolumn{5}{|c|}{ IVNS } \\
\hline & & & & & $f_{\text {best }}$ & $f_{a v g}$ & $f_{\text {worst }}$ & $\sigma$ & time $_{a v g}(s)$ \\
\hline $100 \_15 \_270001$ & 19000 & 19174 & 19000 & 19000 & 19000 & 19000.00 & 19000 & 0.00 & 1.01 \\
\hline $100 \_15 \_270002$ & 22686 & 22686 & 22686 & 23288 & 22686 & 22686.00 & 22686 & 0.00 & 0.71 \\
\hline $100 \_15 \_270003$ & 14558 & 14558 & 14558 & 14616 & 14558 & 14558.00 & 14558 & 0.00 & 0.09 \\
\hline $100 \_15 \_270004$ & 19700 & 19762 & 19700 & 19882 & 19700 & 19700.00 & 19700 & 0.00 & 0.15 \\
\hline $100 \_15 \_270005$ & 22746 & 22892 & 22746 & 23092 & 22746 & 22746.00 & 22746 & 0.00 & 0.67 \\
\hline $100 \_25 \_270001$ & 36412 & 36412 & 36448 & 36752 & 36412 & 36412.00 & 36412 & 0.00 & 2.42 \\
\hline $100 \_25 \_270002$ & 38608 & 39144 & 38608 & 39256 & 38608 & 38608.00 & 38608 & 0.00 & 1.04 \\
\hline $100 \_25 \_270003$ & 32686 & 32966 & 32686 & 32708 & 32686 & 32686.00 & 32686 & 0.00 & 1.82 \\
\hline 100_25_270004 & 35322 & 35678 & 35322 & 35954 & 35322 & 35322.00 & 35322 & 0.00 & 0.26 \\
\hline $100 \_25 \_270005$ & 36878 & 36906 & 36878 & 37100 & 36690 & 36690.00 & 36690 & 0.00 & 0.21 \\
\hline $100 \_50 \_270001$ & 60922 & 60922 & 61172 & 61554 & 60922 & 60922.00 & 60922 & 0.00 & 2.58 \\
\hline $100 \_50 \_270002$ & 62022 & 62046 & 62022 & 62524 & 62022 & 62022.00 & 62022 & 0.00 & 0.53 \\
\hline $100 \_50 \_270003$ & 54596 & 54618 & 54596 & 55192 & 54596 & 54596.00 & 54596 & 0.00 & 4.07 \\
\hline 100_50_270004 & 57894 & 57894 & $\mathbf{5 7 8 9 4}$ & 58208 & 57894 & 57894.00 & 57894 & 0.00 & 1.18 \\
\hline $100 \_50 \_270005$ & 61088 & 61088 & 61318 & 62784 & 61080 & 61082.80 & 61090 & 4.31 & 46.02 \\
\hline $200 \_15 \_270001$ & 81558 & 81558 & 82834 & 81558 & 81558 & 81558.00 & 81558 & 0.00 & 12.53 \\
\hline $200 \_15 \_270002$ & 89810 & 89810 & 90620 & 90506 & 89492 & 90502.80 & 91172 & 546.53 & 48.73 \\
\hline $200 \_15 \_270003$ & 79232 & 79232 & 80980 & 79548 & 79232 & 79277.60 & 80144 & 198.77 & 13.39 \\
\hline $200 \_15 \_270004$ & 78324 & 78324 & 80538 & 80026 & 78324 & 78485.50 & 79726 & 375.08 & 52.95 \\
\hline $200 \_15 \_270005$ & 95998 & 95998 & 98826 & 98830 & 95680 & 96137.10 & 96986 & 622.92 & 21.85 \\
\hline $200 \_25 \_270001$ & 133168 & 133168 & 138454 & 140492 & 133168 & 133168.00 & 133168 & 0.00 & 51.17 \\
\hline $200 \_25 \_270002$ & 136038 & 136038 & 140066 & 140690 & 133778 & 133859.80 & 133926 & 47.19 & 68.02 \\
\hline $200 \_25 \_270003$ & 139438 & 139438 & 144120 & 143724 & 136782 & 136795.50 & 136812 & 14.92 & 67.84 \\
\hline $200 \_25 \_270004$ & 128554 & 128554 & 134054 & 131786 & 128246 & 128246.00 & 128246 & 0.00 & 53.92 \\
\hline $200 \_25 \_270005$ & 148402 & 148402 & 154260 & 152934 & 147844 & 147844.00 & 147844 & 0.00 & 10.20 \\
\hline $200 \_50 \_270001$ & 219672 & 221550 & 223096 & 223098 & 215388 & 215531.20 & 215572 & 64.28 & 67.70 \\
\hline $200-50 \_270002$ & 216444 & 218254 & 219910 & 219834 & 212798 & 212864.60 & 212912 & 36.18 & 92.01 \\
\hline $200 \_50 \_270003$ & 221348 & 221500 & 222404 & 221110 & 214364 & 214413.90 & 214426 & 15.03 & 61.89 \\
\hline $200 \_50 \_270004$ & 211832 & 212044 & 212544 & 213170 & 206476 & 206509.80 & 206590 & 29.01 & 61.66 \\
\hline 200_50_270005 & 231890 & 231890 & 236136 & 237156 & 229918 & 230050.70 & 230082 & 22.82 & 76.55 \\
\hline $400 \_15 \_270001$ & 370314 & 372694 & 456158 & 375650 & 369048 & 372055.40 & 385786 & 4442.20 & 187.66 \\
\hline $400 \_15 \_270002$ & 370274 & 370274 & 460232 & 383096 & 365878 & 369275.90 & 378508 & 4126.82 & 228.23 \\
\hline $400 \_15 \_270003$ & 358684 & 358684 & 448830 & 366314 & 352588 & 356988.80 & 365886 & 4140.92 & 135.41 \\
\hline $400 \_15 \_270004$ & 334430 & 334430 & 406834 & 346282 & 331888 & 339169.90 & 350388 & 6361.04 & 214.63 \\
\hline $400-15 \_270005$ & 361904 & 361904 & 457274 & 377094 & 360422 & 363890.10 & 383154 & 4970.80 & 200.30 \\
\hline $400 \_25 \_270001$ & 568830 & 570852 & 663908 & 579130 & 545118 & 546936.70 & 549318 & 1026.66 & 208.04 \\
\hline $400 \_25 \_270002$ & 543182 & 544568 & 658440 & 554840 & 528470 & 529087.80 & 530118 & 444.68 & 201.85 \\
\hline $400 \_25 \_270003$ & 548000 & 548000 & 667982 & 553162 & 524678 & 526220.60 & 530438 & 1695.07 & 215.17 \\
\hline $400 \_25 \_270004$ & 501750 & 501750 & 607672 & 516416 & 481568 & 482070.00 & 484566 & 722.65 & 174.58 \\
\hline $400 \_25 \_270005$ & 556044 & 556044 & 679848 & 585070 & 548100 & 549839.10 & 557482 & 2608.26 & 240.02 \\
\hline $400 \_50 \_270001$ & 851412 & 851412 & 951882 & 879438 & 824766 & 825581.20 & 826202 & 402.47 & 241.94 \\
\hline $400 \_50 \_270002$ & 845496 & 845496 & 949562 & 874226 & 823094 & 824239.00 & 825442 & 591.82 & 216.54 \\
\hline $400-50 \_270003$ & 819242 & 819242 & 919140 & 843242 & 801586 & 802672.90 & 804310 & 776.71 & 266.22 \\
\hline $400 \_50 \_270004$ & 774564 & 774564 & 878912 & 806690 & 760602 & 761370.40 & 763076 & 553.34 & 275.49 \\
\hline $400 \_50 \_270005$ & 854726 & 854726 & 940358 & 882060 & 828384 & 829335.50 & 830116 & 421.66 & 238.48 \\
\hline \#Improve & 0 & 0 & 0 & 0 & 28 & & & & \\
\hline \#Match & 17 & 9 & 11 & 2 & 17 & & & & \\
\hline \#Total & 45 & 45 & 45 & 45 & 45 & & & & \\
\hline$p$-value & $1.21 \mathrm{e}-7$ & $1.97 \mathrm{e}-9$ & $5.51 \mathrm{e}-9$ & $5.47 \mathrm{e}-11$ & & & & & \\
\hline
\end{tabular}

instance the IVNS algorithm was independently run 20 times. The computational results are summarized in Table 4 for the large instances with $n \geq 100$. For very small instances with $n \leq 40$, the computational results are reported in Appendix (Table 10) since they are very easy to be solved by the IVNS algorithm (see Appendix for the details). Notice that in the present section all results are given in the form of minimization to make a direct comparison between the results of the IVNS algorithm and those reported in the 
literature, and that the results of the maximization form can be converted to the minimization form as follows: $f_{\min }=2\left(\sum_{i<j} c_{i j}-f_{\max }\right)$, where $f_{\min }$ and $f_{\text {max }}$ respectively correspond to the results of minimization and maximization forms.

Columns 1 and 2 of Table 4 respectively give the instance name and the best known solution (BKS) published in the literature. Columns 3-5 show the best results of three reference algorithms in [26]: a GRASP method (GQAP), a GRASP embedded within a population-based evolutionary path-relinking algorithm (GevPR-HMP), and a population-based biased random-key genetic algorithm (BRKGA). The results of these reference algorithms were directly extracted from [26], which correspond to the best outcomes $\left(f_{\text {best }}\right)$ yielded by 5 runs with a cutoff time of 24 hours based on a cluster running Intel X5650 processors at $2.67 \mathrm{GHz}$ or a cluster running Intel Xeon E5530 processors at $2.4 \mathrm{GHz}[26]$. It is worth noting that the cutoff time of the three reference algorithms is much higher than ours (24 hours vs. $n \leq 400$ seconds). Columns 6-10 show the results of our IVNS algorithm, including the best objective value $\left(f_{\text {best }}\right)$ over 20 runs, the average objective value $\left(f_{\text {agv }}\right)$, the worst objective value $\left(f_{\text {worst }}\right)$, the standard deviation of objective value $(\sigma)$, and the average running time in seconds to reach its final objective value $\left(\right.$ time $\left._{\text {avg }}\right)$. The rows Improve, Match denote the number of instances for which the associated algorithm improved or matched the best known results in the literature, and row Total shows the total number of instances. Note that the current best results are indicated in bold, and other symbols are the same as those in Table 2. Besides, it should be mentioned that this section focuses on the best results produced by the compared algorithms, since the compared algorithms were run on different computers and the cut-off times of the reference algorithms are much longer than that of our IVNS algorithm.

Table 4 clearly discloses that the proposed IVNS algorithm outperforms the three reference algorithms designed for the handover minimization problem. First, the IVNS algorithm improved the best known results for 28 out of 45 instances with $n \geq 100$, while matching the best known results for the remaining instances. Second, compared to any of the three reference algorithms, our IVNS algorithm obtained the better or equal objective values for all instances, even if IVNS uses much shorter cutoff times than that of the reference algorithms ( $n \leq 400$ seconds vs. 24 hours). Third, even the worst objective value produced by the IVNS algorithm is better than the best known result reported in the literature for instances with $n=400$, and the average computing time time $_{\text {avg }}$ is smaller than 300 for each instance. Finally, one observes that all p-values are smaller than 0.05 , implying there exits a significant difference between the results of the IVNS algorithm and those yielded by the reference algorithms. In summary, these outcomes indicate that the proposed IVNS algorithm is highly efficient for solving the handover minimization instances compared to the state-of-the-art algorithms in the literature [26]. 


\section{Analysis and Discussions}

We now turn our attention to analyze some essential aspects of the proposed IVNS algorithm, including the local optimization procedure (i.e., the EVND method), the influence of the diversification stage on the performance of IVNS algorithm, and a sensitivity analysis of the key parameters. In this section, all experiments were carried out based on the set of RanReal instances (20 instances with $n=240$ and 20 instances with $n=480$ ).

\subsection{Comparison Between the Standard and Extended VND Methods}

The IVNS algorithm employs the extended VND method (EVND) as its local optimization procedure. Since the EVND method is an extension of the standard VND method, we carried out an experiment to compare both methods. In this experiment, both EVND and VND were respectively run 100 times on each instance. Specifically, for each run, both methods were performed with the same initial solution generated by the first construction procedure presented in Section 2.3.

The computational results of this experiment are summarized in Table 5, including the average objective function value $\left(f_{\text {avg }}\right)$ and the average running time $\left(\right.$ time $\left._{\text {avg }}\right)$. In addition, the rows Better, Equal and Worse of the table denote the number of instances for which the corresponding algorithm obtained a better, equal, and worse average objective value compared to another one. The $p$-values from the non-parametric Friedman test are given in the last row of the table.

It can be observed from Table 5 that in terms of the average objective value, the EVND method achieves a better result than the standard VND method for 36 out of 40 instances, whereas both methods consumed a similar computational time for most instances. These outcomes demonstrate the interest of the EVND method compared to the standard VND method.

\subsection{Importance of 2-1 Exchange Neighborhood $N_{3}$}

The EVND method employs three complementary neighborhoods i.e., $N_{1}, N_{2}$ and the 2-1 exchange neighborhood $N_{3}$. While $N_{1}$ and $N_{2}$ are very popular and their effectiveness has been shown on a number of the clustering problems in the literature $[5,6,9,28,29,31], N_{3}$ is not well studied and thus less understood. In this section, we assess the influence of $N_{3}$ on the performance of the IVNS algorithm. The computational experiment was carried out as follows. 
Table 5

Comparison between the standard VND method and the extended VND (EVND) method on the set of 40 representative instances. Each instance was independently solved 100 times by both algorithms respectively, and better results in the average objective value $\left(f_{\text {avg }}\right)$ between the compared algorithms are indicated in bold.

\begin{tabular}{|c|c|c|c|c|}
\hline \multirow[t]{2}{*}{ Instance } & \multicolumn{2}{|c|}{$f_{a v g}$} & \multicolumn{2}{|c|}{ time $_{a v g}$} \\
\hline & VND & EVND & VND & EVND \\
\hline RanReal240_01 & 220221.45 & 221035.18 & 0.12 & 0.12 \\
\hline RanReal240- 02 & 199165.17 & 199461.37 & 0.09 & 0.10 \\
\hline RanReal240-03 & 193878.39 & 194087.07 & 0.09 & 0.10 \\
\hline RanReal240_04 & 219242.90 & 220280.46 & 0.08 & 0.11 \\
\hline RanReal240־05 & 190443.88 & 190569.72 & 0.08 & 0.10 \\
\hline RanReal240_06 & 211538.07 & 212198.32 & 0.09 & 0.10 \\
\hline RanReal240־07 & 203850.69 & 204429.68 & 0.10 & 0.13 \\
\hline RanReal240-08 & 200600.21 & 200710.54 & 0.13 & 0.12 \\
\hline RanReal240_09 & 204291.07 & 204961.02 & 0.08 & 0.10 \\
\hline RanReal240_10 & 186995.53 & 187202.99 & 0.09 & 0.11 \\
\hline RanReal240- 11 & 199062.90 & 199574.51 & 0.09 & 0.11 \\
\hline RanReal240_12 & 196535.07 & 196618.48 & 0.08 & 0.11 \\
\hline RanReal240 13 & 197326.13 & 197585.12 & 0.10 & 0.11 \\
\hline RanReal240_14 & 224438.53 & 224784.93 & 0.12 & 0.12 \\
\hline RanReal240_15 & 185489.25 & 186227.47 & 0.08 & 0.10 \\
\hline RanReal240 16 & 198794.30 & 199277.26 & 0.10 & 0.11 \\
\hline RanReal240_17 & 189651.07 & 190188.43 & 0.07 & 0.10 \\
\hline RanReal240-18 & 189290.28 & 189691.65 & 0.10 & 0.10 \\
\hline RanReal240 19 & 193267.47 & 194274.68 & 0.08 & 0.10 \\
\hline RanReal240_20 & 207193.59 & 207692.77 & 0.08 & 0.10 \\
\hline RanReal480 01 & 541860.57 & 545446.40 & 0.88 & 0.87 \\
\hline RanReal480־02 & 497118.68 & 498148.99 & 1.01 & 0.95 \\
\hline RanReal480_03 & 483285.49 & 482184.26 & 0.99 & 0.99 \\
\hline RanReal480-04 & 507951.82 & 509674.81 & 0.76 & 0.90 \\
\hline RanReal480־05 & 469493.30 & 469172.22 & 0.86 & 0.91 \\
\hline RanReal480_06 & 516732.69 & 518796.77 & 0.96 & 0.95 \\
\hline RanReal480- 07 & 528136.56 & 533541.23 & 0.99 & 0.93 \\
\hline RanReal480_08 & 516413.17 & 518435.89 & 0.81 & 0.83 \\
\hline RanReal480-09 & 543150.66 & 546057.68 & 0.88 & 0.98 \\
\hline RanReal480-10 & 507686.31 & 508665.48 & 0.77 & 0.86 \\
\hline RanReal480_11 & 511503.09 & 512682.60 & 0.96 & 0.94 \\
\hline RanReal480 12 & 487411.85 & 488100.45 & 1.04 & 1.08 \\
\hline RanReal480_13 & 517853.71 & 521632.82 & 0.87 & 0.88 \\
\hline RanReal480 14 & 500399.35 & 500139.40 & 1.01 & 0.94 \\
\hline RanReal480- 15 & 501547.03 & 503165.49 & 0.88 & 0.83 \\
\hline RanReal480_16 & 536611.41 & 537921.48 & 1.00 & 0.86 \\
\hline RanReal480 17 & 526315.11 & 526884.43 & 0.92 & 0.95 \\
\hline RanReal480_18 & 508572.09 & 511441.64 & 0.88 & 0.85 \\
\hline RanReal480_19 & 509748.10 & 509208.68 & 0.70 & 0.84 \\
\hline RanReal480_20 & 502534.92 & 504453.71 & 0.93 & 0.87 \\
\hline \#Better & 4 & 36 & & \\
\hline \#Equal & 0 & 0 & & \\
\hline \#Worse & 36 & 4 & & \\
\hline$p$-value & & $4.20 \mathrm{e}-7$ & & $1.96 \mathrm{e}-2$ \\
\hline
\end{tabular}

We ran our IVNS and IVNS ${ }^{-}$methods 20 times to solve each instance, where IVNS $^{-}$is a variant of IVNS in which $N_{3}$ (corresponding to subroutine $L S N_{3}$ of Algorithm 6) is disabled while keeping the other algorithmic ingredients unchanged. The experimental results are summarized in Table 6, including the average objective value $f_{\text {avg }}$, the standard deviation of objective value $(\sigma)$, and the average running time to reach its final objective value $\left(\right.$ time $\left._{\text {avg }}\right)$, and other symbols are the same as those in the previous tables.

Table 6 shows that without $N_{3}$, the performance of IVNS deteriorates for all instances in terms of average objective value. Moreover, the average computing times indicate that $N_{3}$ helps the IVNS algorithm to continue its search for a 
Table 6

Comparison between the IVNS method and its a variant $\left(\mathrm{IVNS}^{-}\right)$in which the neighborhood $N_{3}$ is disabled on the set of 40 representative instances. Each instance is respectively solved 20 times by both algorithms, and better results in the average objective value between two algorithms are indicated in bold.

\begin{tabular}{|c|c|c|c|c|c|c|}
\hline \multirow[t]{2}{*}{ Instance } & \multicolumn{2}{|c|}{$f_{\text {avg }}$} & \multicolumn{2}{|c|}{$\sigma$} & \multicolumn{2}{|c|}{ time $_{a v g}$} \\
\hline & IVNS $^{-}$ & IVNS & IVNS $^{-}$ & IVNS & IVNS $^{-}$ & IVNS \\
\hline $\begin{array}{ll}\text { RanReal240 } & 01\end{array}$ & 223891.05 & 224785.27 & 179.47 & 97.88 & 114.90 & 147.28 \\
\hline RanReal240- 02 & 203789.58 & 204415.88 & 160.64 & 102.77 & 120.46 & 160.27 \\
\hline RanReal240_03 & 198236.57 & 198626.93 & 96.21 & 170.83 & 145.08 & 146.88 \\
\hline RanReal240 04 & 224617.33 & 225227.11 & 86.58 & 237.09 & 149.30 & 162.63 \\
\hline RanReal240- 05 & 195181.62 & 195228.86 & 82.96 & 91.62 & 149.44 & 161.77 \\
\hline RanReal240_06 & 215491.95 & 216474.84 & 126.77 & 169.61 & 109.82 & 165.88 \\
\hline RanReal240-07 & 208889.10 & 209004.05 & 74.03 & 120.82 & 147.34 & 120.41 \\
\hline RanReal240-08 & 204207.83 & 204958.19 & 230.94 & 139.71 & 158.62 & 174.29 \\
\hline RanReal240- 09 & 208562.04 & 208789.79 & 50.70 & 148.77 & 119.07 & 160.17 \\
\hline RanReal240-10 & 192253.79 & 192788.59 & 100.09 & 154.18 & 147.11 & 170.78 \\
\hline RanReal240-11 & 203776.92 & 204523.95 & 109.20 & 96.57 & 149.70 & 148.56 \\
\hline RanReal240- 12 & 200251.35 & 200904.16 & 145.50 & 126.79 & 101.56 & 169.33 \\
\hline RanReal240- 13 & 201581.76 & 202139.55 & 143.75 & 155.41 & 123.42 & 150.75 \\
\hline RanReal240-14 & 228332.63 & 228512.11 & 228.63 & 170.71 & 108.74 & 141.27 \\
\hline RanReal240 15 & 190130.92 & 190914.31 & 190.24 & 174.08 & 137.21 & 144.95 \\
\hline RanReal240_16 & 203072.49 & 203834.68 & 197.23 & 131.32 & 110.16 & 147.86 \\
\hline RanReal240_17 & 194564.42 & 195114.49 & 92.24 & 109.08 & 113.22 & 146.03 \\
\hline RanReal240- 18 & 194370.83 & 194853.70 & 121.64 & 126.17 & 107.11 & 163.49 \\
\hline RanReal240_19 & 198496.90 & 199019.23 & 144.56 & 109.79 & 111.23 & 176.31 \\
\hline RanReal240-20 & 211418.56 & 212046.92 & 130.93 & 130.92 & 106.77 & 149.66 \\
\hline RanReal480- 01 & 550160.70 & 554331.89 & 777.24 & 415.49 & 304.77 & 369.26 \\
\hline RanReal480_02 & 507893.39 & 509519.84 & 457.07 & 569.71 & 286.47 & 416.40 \\
\hline RanReal480-03 & 493226.43 & 495847.80 & 584.05 & 426.86 & 307.52 & 415.80 \\
\hline RanReal480-04 & 518088.82 & 520891.75 & 462.43 & 648.97 & 303.57 & 380.20 \\
\hline RanReal480_05 & 481778.58 & 482595.19 & 436.13 & 497.25 & 310.42 & 338.96 \\
\hline RanReal480-06 & 529846.53 & 532888.64 & 537.12 & 555.89 & 240.48 & 338.25 \\
\hline RanReal480_07 & 542575.23 & 544530.14 & 675.84 & 413.13 & 310.92 & 388.74 \\
\hline RanReal480-08 & 529264.25 & 531417.94 & 464.02 & 555.42 & 265.15 & 373.48 \\
\hline RanReal480_09 & 551329.66 & 555098.72 & 547.85 & 514.64 & 246.68 & 388.08 \\
\hline RanReal480_10 & 516929.26 & 518612.02 & 466.20 & 589.69 & 282.97 & 403.46 \\
\hline RanReal480 11 & 520357.01 & 522814.96 & 424.04 & 402.37 & 318.02 & 382.52 \\
\hline RanReal480_12 & 498472.55 & 500580.84 & 410.95 & 540.16 & 258.80 & 386.04 \\
\hline RanReal480_13 & 530678.39 & 533763.20 & 628.41 & 423.89 & 261.10 & 420.59 \\
\hline RanReal480 14 & 509895.03 & 512975.73 & 685.12 & 408.22 & 251.09 & 401.09 \\
\hline RanReal480_15 & 513947.87 & 516017.98 & 645.76 & 408.07 & 255.18 & 375.96 \\
\hline RanReal480_16 & 544921.69 & 548276.15 & 933.12 & 590.34 & 332.77 & 366.12 \\
\hline RanReal480- 17 & 533634.44 & 536655.06 & 498.31 & 402.38 & 277.45 & 389.54 \\
\hline RanReal480_18 & 521497.91 & 524650.86 & 775.43 & 494.86 & 288.94 & 379.04 \\
\hline RanReal480 19 & 519184.62 & 521180.84 & 716.91 & 550.77 & 230.32 & 405.61 \\
\hline RanReal480_20 & 514755.82 & 517261.92 & 500.77 & 530.58 & 286.77 & 398.10 \\
\hline \#Better & 0 & 40 & & & & \\
\hline \#Equal & 0 & 0 & & & & \\
\hline \#Worse & 40 & 0 & & & & \\
\hline$p$-value & & $2.54 \mathrm{e}-10$ & & & & \\
\hline
\end{tabular}

longer time and thus to attain better solutions. This experiment demonstrates the usefulness of the 2-1 exchange neighborhood for the IVNS algorithm.

\subsection{Importance of the Diversification Mechanism}

The IVNS algorithm performs an intensified search stage with the iterated local optimization (lines 5-17 of Algorithm 1) and a diversified stage with the Shake procedure (line 18 of Algorithm 1). The diversified stage aims at producing transition states between two high-quality local optima, since these transition states are usually necessary to help the search process to move from 
Table 7

Comparative results of the IVNS method with and without its diversified stage (IVNS-D), on the set of 40 representative instances. Each instance was independently solved 20 times by both algorithms respectively, and better results in terms of the average objective value between two algorithms are indicated in bold.

\begin{tabular}{|c|c|c|c|c|c|c|}
\hline \multirow[t]{2}{*}{ Instance } & \multicolumn{2}{|c|}{$f_{a v g}$} & \multicolumn{2}{|c|}{$\sigma$} & \multicolumn{2}{|c|}{ time $_{a v g}$} \\
\hline & IVNS-D & IVNS & IVNS-D & IVNS & IVNS-D & IVNS \\
\hline RanReal240 01 & 223986.99 & 224785.27 & 272.57 & 97.88 & 69.37 & 147.28 \\
\hline RanReal240_02 & 203614.45 & 204415.88 & 299.81 & 102.77 & 113.36 & 160.27 \\
\hline RanReal240־03 & 197731.77 & 198626.93 & 418.98 & 170.83 & 100.27 & 146.88 \\
\hline RanReal240־04 & 224424.68 & 225227.11 & 459.32 & 237.09 & 93.67 & 162.63 \\
\hline RanReal240_05 & 194298.12 & 195228.86 & 474.64 & 91.62 & 113.46 & 161.77 \\
\hline RanReal240_06 & 215609.74 & 216474.84 & 318.55 & 169.61 & 90.56 & 165.88 \\
\hline RanReal240_07 & 208341.50 & 209004.05 & 378.79 & 120.82 & 122.25 & 120.41 \\
\hline RanReal240_08 & 204211.41 & 204958.19 & 219.57 & 139.71 & 93.92 & 174.29 \\
\hline RanReal240_09 & 208092.99 & 208789.79 & 286.18 & 148.77 & 109.41 & 160.17 \\
\hline RanReal240_10 & 191828.97 & 192788.59 & 482.02 & 154.18 & 133.85 & 170.78 \\
\hline RanReal240_11 & 203921.95 & 204523.95 & 329.82 & 96.57 & 75.89 & 148.56 \\
\hline RanReal240-12 & 199971.56 & 200904.16 & 300.91 & 126.79 & 105.04 & 169.33 \\
\hline RanReal240_13 & 201224.78 & 202139.55 & 467.84 & 155.41 & 87.82 & 150.75 \\
\hline RanReal240 14 & 227825.89 & 228512.11 & 356.96 & 170.71 & 103.34 & 141.27 \\
\hline RanReal240-15 & 189814.49 & 190914.31 & 446.62 & 174.08 & 81.73 & 144.95 \\
\hline RanReal240_16 & 202951.20 & 203834.68 & 412.54 & 131.32 & 87.63 & 147.86 \\
\hline RanReal240 17 & 194328.55 & 195114.49 & 265.17 & 109.08 & 165.12 & 146.03 \\
\hline RanReal240_18 & 193915.24 & 194853.70 & 286.06 & 126.17 & 104.30 & 163.49 \\
\hline RanReal240_19 & 197900.00 & 199019.23 & 461.25 & 109.79 & 101.25 & 176.31 \\
\hline RanReal240_20 & 211284.31 & 212046.92 & 280.17 & 130.92 & 96.18 & 149.66 \\
\hline RanReal480_01 & 552979.67 & 554331.89 & 560.30 & 415.49 & 375.66 & 369.26 \\
\hline RanReal480-02 & 507974.23 & 509519.84 & 816.89 & 569.71 & 337.88 & 416.40 \\
\hline RanReal480_03 & 494000.83 & 495847.80 & 961.92 & 426.86 & 372.23 & 415.80 \\
\hline RanReal480_04 & 519475.06 & 520891.75 & 843.47 & 648.97 & 367.62 & 380.20 \\
\hline RanReal480_05 & 481098.07 & 482595.19 & 832.34 & 497.25 & 315.49 & 338.96 \\
\hline RanReal480_06 & 531667.60 & 532888.64 & 649.14 & 555.89 & 360.97 & 338.25 \\
\hline RanReal480_07 & 543160.19 & 544530.14 & 666.03 & 413.13 & 345.01 & 388.74 \\
\hline RanReal480_08 & 530127.30 & 531417.94 & 626.52 & 555.42 & 369.13 & 373.48 \\
\hline RanReal480_09 & 553832.78 & 555098.72 & 703.40 & 514.64 & 383.55 & 388.08 \\
\hline RanReal480_10 & 516798.04 & 518612.02 & 653.19 & 589.69 & 327.60 & 403.46 \\
\hline RanReal480-11 & 520835.32 & 522814.96 & 952.45 & 402.37 & 359.04 & 382.52 \\
\hline RanReal480_12 & 499078.98 & 500580.84 & 687.26 & 540.16 & 334.42 & 386.04 \\
\hline RanReal480_13 & 532308.08 & 533763.20 & 792.43 & 423.89 & 390.62 & 420.59 \\
\hline RanReal480-14 & 511808.09 & 512975.73 & 635.87 & 408.22 & 408.37 & 401.09 \\
\hline RanReal480_15 & 514720.84 & 516017.98 & 482.10 & 408.07 & 365.89 & 375.96 \\
\hline RanReal480_16 & 547296.05 & 548276.15 & 682.57 & 590.34 & 371.18 & 366.12 \\
\hline RanReal480_17 & 534975.71 & 536655.06 & 719.18 & 402.38 & 353.90 & 389.54 \\
\hline RanReal480 18 & 523253.63 & 524650.86 & 720.31 & 494.86 & 348.96 & 379.04 \\
\hline RanReal480_19 & 519700.55 & 521180.84 & 564.17 & 550.77 & 378.77 & 405.61 \\
\hline RanReal480_20 & 515947.56 & 517261.92 & 567.91 & 530.58 & 325.18 & 398.10 \\
\hline \#Better & 0 & 40 & & & & \\
\hline \#Equal & 0 & 0 & & & & \\
\hline \#Worse & 40 & 0 & & & & \\
\hline$p$-value & & $2.54 \mathrm{e}-10$ & & & & \\
\hline
\end{tabular}

a basin of attraction to another basin.

In order to assess the impact of this diversified stage on the performance of the IVNS algorithm, we created a variant of the IVNS method (denoted by IVNS-D) by removing the Shake operation of line 18 of Algorithm 1 while keeping other components of IVNS unchanged. We ran IVNS-D and IVNS 20 times to solve each instance. The experimental results are summarized in Table 7, where the symbols have the same meanings as those in the previous tables.

Table 7 indicates that IVNS-D deteriorates the results of IVNS. First, IVNS-D performs worse than IVNS on all instances in terms of the average objective 
557 value. Second, concerning the standard deviation $(\sigma)$ of the objective value, 558 IVNS obtained a better result for all instances. This experiment confirms 559 the usefulness of the additional diversification stage introduced in line 18 of

560 Algorithm 1.

\subsection{Sensitivity Analysis of Parameters}

Table 8

Sensitivity analysis of the parameter $m$. Each instance was independently solved 20 times by the IVNS algorithm for each parameter value in the range $\{4,6,8,10,12,14,16,18\}$, and the average objective values $\left(f_{\text {avg }}\right)$ over 20 runs are respectively reported.

\begin{tabular}{|c|c|c|c|c|c|c|c|c|}
\hline \multirow[b]{2}{*}{ Instance $/ m$} & \multicolumn{8}{|c|}{$f_{a v g}$} \\
\hline & 4 & 6 & 8 & 10 & 12 & 14 & 16 & 18 \\
\hline $\begin{array}{ll}\text { RanReal240 } & 01\end{array}$ & 224747.89 & 224743.77 & 224750.51 & 224736.19 & 224734.17 & 224784.25 & 224725.39 & 224718.09 \\
\hline RanReal240 02 & 204400.52 & 204403.15 & 204425.39 & 204459.24 & 204400.94 & 204418.82 & 204448.37 & 204449.36 \\
\hline RanReal240_03 & 198657.99 & 198717.34 & 198685.34 & 198691.63 & 198679.41 & 198658.77 & 198661.33 & 198685.05 \\
\hline RanReal240_04 & 225204.66 & 225140.01 & 225146.45 & 225190.13 & 225152.00 & 225194.58 & 225242.84 & 225239.61 \\
\hline RanReal240_05 & 195275.00 & 195287.90 & 195237.80 & 195259.40 & 195256.56 & 195199.85 & 195246.73 & 195246.16 \\
\hline RanReal240_06 & 216533.35 & 216475.23 & 216454.28 & 216497.78 & 216513.23 & 216465.82 & 216515.60 & 216491.18 \\
\hline RanReal240_07 & 209098.93 & 209062.00 & 209060.14 & 2090 & 2090 & 2090 & 209 & 9.07 \\
\hline RanReal240_08 & 204914.37 & 204947 & 2048 & 2049 & 2049 & 2049 & 204 & 2049 \\
\hline RanReal240 09 & 208826.26 & 208832.55 & 208883 & 208833.99 & 20879 & 20879 & 2088 & 208745.94 \\
\hline RanReal240 10 & 192736.25 & 192755.01 & 192815.78 & 192698.13 & 192811.66 & 1927 & 1926 & 192796.48 \\
\hline RanReal240 11 & 204478.83 & 204444.22 & 204464.45 & 204448.14 & 2044 & 2044 & 204 & 204454.86 \\
\hline RanReal240 12 & 200921.94 & 2008 & 200 & 2008 & 200 & 200 & 200 & 200824.69 \\
\hline RanReal240_13 & 202 & 202 & 20 & 202 & 20 & & 20 & 98.55 \\
\hline $40^{-} 14$ & 228 & & & 88 & & & & 7.20 \\
\hline RanReal240_15 & 190924.82 & $190 s$ & & 90 & 190 & 190 & & 3.93 \\
\hline RanReal240_16 & 203763.28 & 2037 & 2037 & 2038 & 2038 & 203 & 2037 & 203801.18 \\
\hline RanReal240_17 & 195115.48 & 1951 & 1951 & 1951 & 195 & .20 & 195 & 195141.27 \\
\hline RanReal240_18 & 194947.69 & 194852.90 & 1948 & 194756.48 & 194875.62 & 2.77 & 1948 & 194836.40 \\
\hline RanReal240_19 & 198962.52 & 199040.68 & 198966.06 & 198984.75 & 198934.98 & 199008.06 & 198988.46 & 198974.27 \\
\hline RanReal240_20 & 212074.56 & 212092.28 & 212056.10 & 212032.47 & 211998.49 & 212046.46 & 212001.59 & 212105.61 \\
\hline Average & 205607.69 & 205602.48 & 205596.71 & 205599.85 & 205590.95 & 205598.16 & 205596.71 & 205601.96 \\
\hline
\end{tabular}

Table 9

Sensitivity analysis of the parameter $\beta_{\max }$. Each instance was independently solved 20 times by the IVNS algorithm for each parameter value in the range $\{5,10,15,20,25,30,35,40\}$, and the average objective values $\left(f_{\text {avg }}\right)$ over 20 runs are respectively reported.

\begin{tabular}{|c|c|c|c|c|c|c|c|c|}
\hline \multirow[b]{2}{*}{ Instance $/ \beta_{\max }$} & \multicolumn{8}{|c|}{$f_{\text {avg }}$} \\
\hline & 5 & 10 & 15 & 20 & 25 & 30 & 35 & 40 \\
\hline RanReal240_01 & 224676.47 & 224758.30 & 224738.12 & 224753.19 & 224749.78 & 224769.53 & 224739.29 & 224675.94 \\
\hline RanReal240_02 & 204384.58 & 204428.48 & 204454.16 & 204422.07 & 204403.51 & 204425.57 & 204381.89 & 204378.07 \\
\hline RanReal240_03 & 198621.22 & 198612.88 & 198694.68 & 198622.62 & 198700.29 & 198600.93 & 198693.71 & 198489.30 \\
\hline RanReal240_04 & 225111.94 & 225215.51 & 225217.08 & 225262.81 & 225203.17 & 225225.99 & 225039.70 & 225189.87 \\
\hline RanReal240_05 & 195202.16 & 195260.55 & 195222.28 & 195278.64 & 195256.13 & 195158.88 & 195199.11 & 195273.42 \\
\hline RanReal240_06 & 216421.63 & 216540.77 & 216524.29 & 216511.22 & 216500.76 & 216531.96 & 216491.43 & 216397.47 \\
\hline RanReal240_07 & 208954.86 & 209051.89 & 209053.73 & 209066.47 & 209030.62 & 209042.97 & 209025.39 & 208978.27 \\
\hline RanReal240_08 & 204957.91 & 204935.06 & 204950.87 & 204998.23 & 204918.30 & 204968.93 & 204864.60 & 204754.79 \\
\hline RanReal240_09 & 208775.98 & 208872.97 & 208803.31 & 208762.89 & 208814.66 & 208793.45 & 208786.65 & 208819.28 \\
\hline RanReal240_10 & 192729.71 & 192761.86 & 192767.85 & 192774.27 & 192787.08 & 192666.16 & 192647.02 & 192789.16 \\
\hline RanReal240_11 & 204457.23 & 204464.00 & 204483.40 & 204492.54 & 204490.35 & 204487.99 & 204463.07 & 204385.61 \\
\hline RanReal240_12 & 200897.62 & 200913.55 & 200930.03 & 200918.37 & 200868.58 & 200824.06 & 200865.56 & 200857.78 \\
\hline RanReal240_13 & 202110.38 & 202121.17 & 202114.62 & 202100.76 & 202097.89 & 202124.86 & 202087.71 & 202049.26 \\
\hline RanReal240_14 & 228454.78 & 228514.51 & 228484.05 & 228516.60 & 228536.93 & 228490.37 & 228494.82 & 228413.24 \\
\hline RanReal240_15 & 190869.97 & 190940.52 & 190895.56 & 190944.22 & 190966.06 & 190940.83 & 190868.54 & 190804.69 \\
\hline RanReal240_16 & 203702.06 & 203824.85 & 203821.77 & 203859.91 & 203778.12 & 203774.10 & 203819.94 & 203790.17 \\
\hline RanReal240_17 & 195096.28 & 195211.71 & 195161.74 & 195155.80 & 195166.45 & 195159.81 & 195051.22 & 194973.69 \\
\hline RanReal240_18 & 194861.25 & 194879.05 & 194882.91 & 194900.61 & 194900.42 & 194861.18 & 194881.60 & 194746.11 \\
\hline RanReal240_19 & 199005.21 & 199037.12 & 199047.45 & 198990.69 & 198983.59 & 199071.70 & 198893.53 & 198805.32 \\
\hline RanReal240_20 & 211997.53 & 212060.94 & 212059.02 & 212049.21 & 212060.15 & 212052.58 & 211986.43 & 211926.56 \\
\hline Average & 205564.44 & 205620.28 & 205615.35 & 205619.06 & 205610.64 & 205598.59 & 205564.06 & 205524.90 \\
\hline
\end{tabular}

${ }_{562}$ Our IVNS algorithm employs two main parameters, i.e., $m$ and $\beta_{\max }$. Pa- 
rameter $m$ is employed in the EVND procedure (Section 2.4.3) to control the exploitation balance between the different neighborhoods, a larger value of $m$ leading to a more balanced neighborhood exploitation. Parameter $\beta_{\max }$ is used to control the strength of intensification search, a larger value of $\beta_{\text {max }}$ implying a stronger intensification for the IVNS algorithm. In this section we show a sensitivity analysis of these two key parameters, which also helps to find an appropriate value for each of them.

In this study, we carried out two additional experiments based on 20 RanReal instances with $n=240$. In the first experiment, we varied the value of $m$ within the range $\{4,6,8,10,12,14,16,18\}$ and ran the algorithm 20 times for each value of $m$ and each instance, while keeping other parameters with their default values as shown in Table 1. The computational results are summarized in Table 8, where the second row indicates the values of $m$, the first column gives the names of instances, the other columns show the average objective function values over 20 independent runs $\left(f_{\text {avg }}\right)$ for each value of $m$ and each instance, and the last row shows the average results over all instances. Similarly, we varied in the second experiment the value of $\beta_{\max }$ within the range $\{5,10,15,20,25,30,35,40\}$. The computational results are summarized in Table 9 , where the second row gives the values of $\beta_{\text {max }}$, and the other entries have the same meanings as those in Table 8.

First, we observe from Table 8 that the performance of the IVNS algorithm is not sensitive to the setting of parameter $m$. Specifically, for most instances the different values of $m$ leaded to very similar results in terms of $f_{\text {avg }}$. Indeed, the relative difference between the results yielded by the different parameter values across the 20 instances is very small $\left(\leq \frac{(205607.69-205590.95)}{205607.69} \times 100 \%=0.0081 \%\right)$. Hence, the default value of $m$ was set to 10 in this work. As for $\beta_{\max }$, Table 9 shows that for most instances the tested values leaded also to similar results in terms of $f_{\text {avg }}$, with a very small relative difference between the results yielded by the different $\beta_{\max }$ values $\left(\leq \frac{(205620.28-205524.90)}{205620.28} \times 100 \%=0.046 \%\right)$. These outcomes indicate that the IVNS algorithm is not sensitive to the setting of parameter $\beta_{\max }$. Consequently, to ensure that a lasting intensified search effect when a long computational time is allowed, the default value of $\beta_{\max }$ was set to 30 in this study.

\section{Conclusions}

The capacitated clustering problem (CCP) is a general and useful model for a number of applications. It also generalizes three well-known NP-hard problems: the maximally diverse grouping problem, the graph partitioning problem, and the handover minimization problem. In this paper, we proposed the iterated variable neighborhood search (IVNS) algorithm for solving the CCP. 
The proposed algorithm organically combines an extended variable neighborhood descent (EVND) method for intensification and a shake procedure for diversification.

The proposed algorithm was assessed on the 133 instances commonly used in the literature, and the computational results indicated that our IVNS algorithm significantly outperforms the state-of-the-art CCP algorithms both in terms of solution quality and computational efficiency. In particular, the proposed algorithm improved the best known results (new lower bounds) for 28 out of 83 handover minimization instances, while matching the best known results for the 55 remaining instances.

The investigations of several essential components of the proposed algorithm shed light on the following points. First, for the CCP, the EVND method usually outperforms the standard variable neighborhood descent method in terms of the local search ability, and the 2-1 exchange neighborhood $N_{3}$ reinforces the intensified search capacity of the EVND method. Second, the diversification stage is essential for the proposed algorithm to reach a suitable trade-off between the diversification and intensification of the search process.

Based on this work, we advance some research perspectives for further improvements. First, within the IVNS algorithm, diversification is ensured by the shake procedure as well as the shake strength. Since different degrees of diversification may be needed at different search stages, it would be interesting to investigate adaptive techniques able to adjust dynamically the shake strength. Moreover, to escape deep local optima, it would also be useful to study other diversification methods like random or adaptive restarts. Second, using the presented EVND method as a local optimization procedure, it may be possible to devise more efficient hybrid evolutionary algorithms for the CCP. Third, the IVNS algorithm only visits feasible solutions. Meanwhile, previous studies like $[8,16]$ showed that tunneling through feasible and infeasible regions can improve the performance of the search process. It would be relevant to study dedicated methods able to explore infeasible regions in a controlled manner. Finally, given that the basic idea of the proposed IVNS algorithm, i.e., integrating organically the EVND method with multiple neighborhoods and a diversified shake procedure, is independent of the CCP, it would be interesting to examine its applicability to other grouping or clustering problems.

\section{Acknowledgments}

We are grateful to the reviewers for their valuable comments which helped us to improve the paper. This work is partially supported by the PGMO project (2013-2015, Jacques Hadamard Mathematical Foundation, Paris, France) and 
a post-doc grant (for X.J. Lai) from the Region of Pays de la Loire (France).

\section{References}

[1] Armas J.D., Melián-Batista B., Moreno-Pérez J.A., Brito J., 2015, GVNS for a real-world rich vehicle routing problem with time windows. Engineering Applications of Artificial Intelligence 42, 45-56.

[2] Bader D.A., Meyerhenke, H., Sanders, P., Wagner D. (eds.) 2013, Graph Partitioning and Graph Clustering. 10th DIMACS Implementation Challenge Workshop. February 13-14, 2012. Georgia Institute of Technology, Atlanta, GA. Contemporary Mathematics 588. American Mathematical Society and Center for Discrete Mathematics and Theoretical Computer Science, 2013.

[3] Benlic U., Hao J.K., 2011, A multilevel memetic approach for improving graph k-partitions. IEEE Transactions on Evolutionary Computation 15(5), 624-642.

[4] Benlic U., Hao J.K., 2013, Hybrid metaheuristics for the graph partitioning problem. In Hybrid Metaheuristics. Studies in Computational Intelligence 434, Chapter 6, pages 157-184.

[5] Brimberg J., Mladenović N., Urošević D., 2015, Solving the maximally diverse grouping problem by skewed general variable neighborhood search. Information Sciences 295, 650-675.

[6] Brimberg J., Janićijević S., Mladenović N., Urošević D., 2015, Solving the clique partitioning problem as a maximally diverse grouping problem. Optimization Letters doi:10.1007/s11590-015-0869-4.

[7] Chen Y., Fan Z.P., Ma J., Zeng S., 2011, A hybrid grouping genetic algorithm for reviewier group construction problem. Expert Systems with Applications 38(3), 2401-2411.

[8] Chen Y., Hao J.K., Glover F., 2016, An evolutionary path relinking approach for the quadratic multiple knapsack problem. Knowledge-based Systems 92, 23-34.

[9] Deng Y.M., Bard J.F., 2011, A reactive GRASP with path relinking for capacitated clustering. Journal of Heuristics 17(2), 119-152.

[10] Fan Z.P., Chen Y., Ma J., Zeng S., 2010, A hybrid genetic algorithmic approach to the maximally diverse grouping problem. Journal of the Operational Research Society 62, 92-99.

[11] Feo T., Khellaf M., 1990, A class of bounded approximation algorithms for graph partitioning. Networks 20(2) 181-195.

[12] Ferreira C.E., Martin A, Souza C.C., Weismantel R., Wolsey L.A., 1996, Formulations and valid inequalities for the node capacitated graph partitioning problem. Mathematical Programming 74(3), 247-266. 
[13] Ferreira C.E., Martin A, Souza C.C., Weismantel R., Wolsey L.A., 1998, The node capacitated graph partitioning problem: a computational study. Mathematical Programming 81(2), 229-256.

[14] Gallego M., Laguna M., Martí R., Duarte A., 2013, Tabu search with strategic oscillation for the maximally diverse grouping problem. Journal of the Operational Research Society 64, 724-734.

[15] Galinier P., Boujbel Z., Fernandes M.C., 2011, An efficient memetic algorithm for the graph partitioning problem. Annals of Operations Research 191(1), 1-22.

[16] Glover F., Hao J.K., 2011, The case for strategic oscillation. Annals of Operations Research 183(1), 163-173.

[17] Hansen, P., Mladenović N., Perez, J.A.M., 2010, Variable neighbourhood search: methods and applications. Annals of Operations Research 175, 367-407.

[18] Hendrickson B., Kolda T.G., 2000, Graph partitioning models for parallel computing. Parallel Computing 26(12), 1519-1534.

[19] Johnes J., 2015, Operational Research in education. European Journal of Operational Research 243(3), 683-696.

[20] Johnson E.L., Mehrotra A., Nemhauser G.L., 1993, Min-cut clustering. Mathematical Programming 62(1-3), 133-151.

[21] Lai X.J., Hao J.K., 2016, Iterated maxima search for the maximally diverse grouping problem. European Journal of Operational Research 254(3), 780-800.

[22] Lewis M., Wang H.B., Kochenberger G., 2014, Exact solutions to the capacitated clustering problem: A comparison of two models. Annals of Data Science 1(1), 1523.

[23] Martínez-Gavara A., Campos V., Gallego M., Laguna M., Martí R., 2015, Tabu search and GRASP for the capacitated clustering problem. Computational Optimization and Applications 62(2), 589-607.

[24] Mladenović N., Hansen P., 1997, Variable neighborhood search. Computers $\&$ Operations Research 24(1), 1097-1100.

[25] Mladenović N., Todosijević R., Urošević D., 2016, Less is more: Basic variable neighborhood search for minimum differential dispersion problem. Information Sciences 326, 160-171.

[26] Morán-Mirabal L.F., González-Velarde J.L., Resende M.G.C., Silva R.M.A., 2013, Randomized heuristics for handover minimization in mobility networks. Journal of Heuristics 19(6), 845-880.

[27] Özsoy F.A., Labbé M., 2010, Size-constrained graph partitioning polytopes. Discrete Mathematics 310(24), 3473-3493.

[28] Palubeckis G., Ostreika A., Rubliauskas D., 2015, Maximally diverse grouping: an iterated tabu search approach. Journal of the Operational Research Society 66, 579-592. 
5 [29] Rodriguez F.J., Lozano M., García-Martínez C., González-Barrera J.D., 2013, 716 An artificial bee colony algorithm for the maximally diverse grouping problem. 717 Information Sciences 230(1), 183-196.

[30] Soper A.J., Walshaw C., Cross M., 2004, A combined evolutionary search and multilevel optimization approach to graph-partitioning. Journal of Global Optimization 29(2), 225-241.

[31] Urošević D., 2014, Variable neighborhood search for maximum diverse grouping problem. Yugoslav Journal of Operations Research 24(1), 21-33.

[32] Villegas J.G., Prins C., Prodhon C., Medaglia A.L., Velasco N., 2010, GRASP/VND and multi-start evolutionary local search for the single truck and trailer routing problem with satellite depots. Engineering Applications of Artificial Intelligence 23(5), 780-794.

[33] Weitz R., Lakshminarayan S., 1997, An empirical comparison of heuristic and 728 graph theoretic methods for creating maximally diverse groups, VLSI design, and 729 exam scheduling. Omega 25(4), 473-482. 
Table 10

Appendix: Computational results of IVNS on the small handover minimization instances. The results are given in the form of minimization to make a direct comparison with the best known results in the literature.

\begin{tabular}{|c|c|c|c|c|c|c|}
\hline \multirow[b]{2}{*}{ Instance } & \multirow[b]{2}{*}{$\mathrm{BKS}$} & \multicolumn{5}{|c|}{ IVNS } \\
\hline & & $f_{\text {best }}$ & $f_{a v g}$ & $f_{\text {worst }}$ & $\sigma$ & time $_{\text {avg }}(s)$ \\
\hline $\begin{array}{lll}20 & 5 & 270001\end{array}$ & 540 & 540 & 540.00 & 540 & 0.00 & 0.00 \\
\hline $20 \_5-270002$ & 54 & 54 & 54.00 & 54 & 0.00 & 0.00 \\
\hline $20-5-270003$ & 816 & 816 & 816.00 & 816 & 0.00 & 0.00 \\
\hline $20-5-270004$ & 126 & 126 & 126.00 & 126 & 0.00 & 0.00 \\
\hline $20{ }^{-} 5^{-} 270005$ & 372 & 372 & 372.00 & 372 & 0.00 & 0.00 \\
\hline $20_{-}^{-} 1 \overline{0} \_270001$ & 2148 & 2148 & 2148.00 & 2148 & 0.00 & 0.00 \\
\hline $20 \_10_{-}^{-} 270002$ & 1426 & 1426 & 1426.00 & 1426 & 0.00 & 0.00 \\
\hline $20^{-} 10^{-} 270003$ & 2458 & 2458 & 2458.00 & 2458 & 0.00 & 0.00 \\
\hline $20_{-}^{-} 10_{-}^{-} 270004$ & 1570 & 1570 & 1570.00 & 1570 & 0.00 & 0.00 \\
\hline $30_{-}^{-} 5 \_\overline{2} 70001$ & 772 & 772 & 772.00 & 772 & 0.00 & 0.00 \\
\hline $30 \_5-270002$ & 136 & 136 & 136.00 & 136 & 0.00 & 0.00 \\
\hline $30-5-270003$ & 920 & 920 & 920.00 & 920 & 0.00 & 0.00 \\
\hline $30-5-270004$ & 52 & 52 & 52.00 & 52 & 0.00 & 0.00 \\
\hline $30-5-270005$ & 410 & 410 & 410.00 & 410 & 0.00 & 0.00 \\
\hline $30-1 \overline{0} \quad 270001$ & 3276 & 3276 & 3276.00 & 3276 & 0.00 & 0.00 \\
\hline $30-10^{-}-270002$ & 1404 & 1404 & 1404.00 & 1404 & 0.00 & 0.00 \\
\hline $30_{-}^{-} 10_{-}^{-} 270003$ & 2214 & 2214 & 2214.00 & 2214 & 0.00 & 0.00 \\
\hline $30_{-}^{-} 10_{-}^{-} 270004$ & 2150 & 2150 & 2150.00 & 2150 & 0.00 & 0.00 \\
\hline $30-10^{-}-270005$ & 2540 & 2540 & 2540.00 & 2540 & 0.00 & 0.00 \\
\hline $30_{-}^{-} 15_{-}^{-} 270001$ & 6178 & 6178 & 6178.00 & 6178 & 0.00 & 0.00 \\
\hline $30-15_{-}^{-} 270002$ & 4042 & 4042 & 4042.00 & 4042 & 0.00 & 0.00 \\
\hline $30 \_15_{-}^{-} 270003$ & 4126 & 4126 & 4126.00 & 4126 & 0.00 & 0.00 \\
\hline $30-15^{-}-270004$ & 3920 & 3920 & 3920.00 & 3920 & 0.00 & 0.00 \\
\hline $40_{-}^{-} 5 \_\overline{2} 70001$ & 610 & 610 & 610.00 & 610 & 0.00 & 0.00 \\
\hline $40 \_5-270002$ & 136 & 136 & 136.00 & 136 & 0.00 & 0.00 \\
\hline $40_{-}^{-} 5_{-}^{-} 270003$ & 234 & 234 & 234.00 & 234 & 0.00 & 0.00 \\
\hline $40-5-270004$ & 232 & 232 & 232.00 & 232 & 0.00 & 0.02 \\
\hline $40_{-}^{-} 5_{-}^{-} 270005$ & 774 & 774 & 774.00 & 774 & 0.00 & 0.00 \\
\hline $40_{-}^{-} 1 \overline{0} \_270001$ & 4544 & 4544 & 4544.00 & 4544 & 0.00 & 0.00 \\
\hline $40_{-}^{-} 10_{-}^{-} 270002$ & 2068 & 2068 & 2068.00 & 2068 & 0.00 & 0.00 \\
\hline $40^{-} 10^{-} 270003$ & 2090 & 2090 & 2090.00 & 2090 & 0.00 & 0.00 \\
\hline $40_{-}^{-} 10_{-}^{-} 270004$ & 1650 & 1650 & 1650.00 & 1650 & 0.00 & 0.01 \\
\hline $40_{-}^{-} 10_{-}^{-} 270005$ & 4316 & 4316 & 4316.00 & 4316 & 0.00 & 0.00 \\
\hline $40_{-}^{-} 15_{-}^{-} 270001$ & 8646 & 8646 & 8646.00 & 8646 & 0.00 & 0.01 \\
\hline $40^{-} 15^{-}-270002$ & 4586 & 4586 & 4586.00 & 4586 & 0.00 & 0.03 \\
\hline $40_{-}^{-} 15_{-}^{-} 270003$ & 5396 & 5396 & 5396.00 & 5396 & 0.00 & 0.01 \\
\hline $40_{-}^{-} 15_{-}^{-} 270004$ & 4800 & 4800 & 4800.00 & 4800 & 0.00 & 0.00 \\
\hline $40_{-}^{-15}-270005$ & 6272 & 6272 & 6272.00 & 6272 & 0.00 & 0.00 \\
\hline
\end{tabular}

